\title{
Symplectic functional analysis and spectral invariants
}

\author{
Booss-Bavnbek, Bernhelm
}

Publication date:
1999

Document Version

Publisher's PDF, also known as Version of record

Citation for published version (APA):

Booss-Bavnbek, B. (1999). Symplectic functional analysis and spectral invariants. Roskilde Universitet. Tekster fra IMFUFA No. 361 http://milne.ruc.dk/ImfufaTekster/

\section{General rights}

Copyright and moral rights for the publications made accessible in the public portal are retained by the authors and/or other copyright owners and it is a condition of accessing publications that users recognise and abide by the legal requirements associated with these rights.

- Users may download and print one copy of any publication from the public portal for the purpose of private study or research. - You may not further distribute the material or use it for any profit-making activity or commercial gain.

- You may freely distribute the URL identifying the publication in the public portal.

\section{Take down policy}

If you believe that this document breaches copyright please contact rucforsk@kb.dk providing details, and we will remove access to the work immediately and investigate your claim. 


\section{TEKST NR 361 \\ 1999}

Symplectic Functional

Analysis and Spectral

Invariants

Bernhelm Booß-Bavnbek

Kenro Furutani

\section{TEKSTER fra}

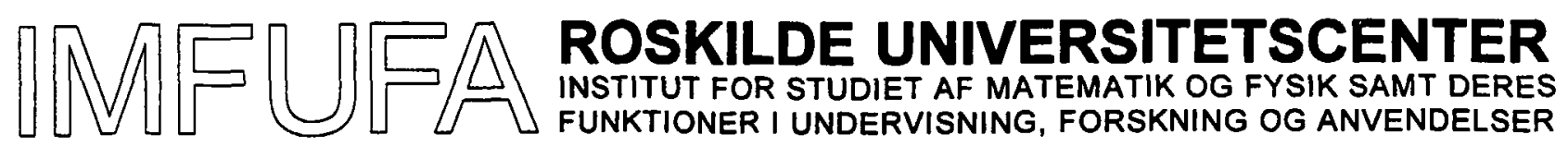


IMFUFA, Roskilde University, P.O.Box 260, 4000 Roskilde, Denmark DEPARTMENT OF STUDIES IN MATHEMATICS AND PHYSICS AND THEIR FUNCTIONS IN EDUCATION, RESEARCH, AND APPLICATIONS

\section{Symplectic Functional Analysis and Spectral Invariants Bernhelm Booß-Bavnbek, Kenro Furutani}

First, we discuss the topology of the Fredholm Lagrangian Grassmannian in a symplectic Hilbert space and show under which conditions the Hörmander index of four Lagrangian subspaces is well defined. As an example, we consider a general spectral flow formula in the symplectic Hilbert space of abstract boundary data for self-adjoint Fredholm extensions of a given 1-parameter family of symmetric operators. We show that the error term, appearing when one replaces one admissible domain by another, equals the Hörmander index.

Then we discuss families of linear elliptic differential operators of first order on a compact manifold $M$ with boundary $\Sigma$. Our framework covers also related questions arising from closed manifolds with fixed hypersurface. We describe the embedding of the symplectic space of abstract boundary data into the distribution space $H^{1 / 2}(\Sigma)$ on the hypersurface and obtain various generalizations of the Yoshida-Nicolaescu spectral flow formula without the assumptions of product structure near the boundary, differentiability of the path, invertibility at the endpoints, and simplicity of the 0-eigenvalues. 


\title{
SYMPLECTIC FUNCTIONAL ANALYSIS AND SPECTRAL INVARIANTS
}

\author{
B. BOOSS-BAVNBEK AND K. FURUTANI
}

\begin{abstract}
First, we discuss the topology of the Fredholm Lagrangian Grassmannian in a symplectic Hilbert space and show under which conditions the Hörmander index of four Lagrangian subspaces is well defined. As an example, we consider a general spectral flow formula in the symplectic Hilbert space of abstract boundary data for self-adjoint Fredholm extensions of a given 1parameter family of symmetric operators. We show that the error term, appearing when one replaces one admissible domain by another, equals the Hörmander index.

Then we discuss families of linear elliptic differential operators of first order on a compact manifold $M$ with boundary $\Sigma$. Our framework covers also related questions arising from closed manifolds with fixed hypersurface. We describe the embedding of the symplectic space of abstract boundary data into the distribution space $H^{-\frac{1}{2}}(\Sigma)$ on the hypersurface and obtain various generalizations of the Yoshida-Nicolaescu spectral flow formula without the assumptions of product structure near the boundary, differentiability of the path, invertibility at the endpoints, and simplicity of the 0-eigenvalues.
\end{abstract}

1991 Mathematics Subject Classification. 58G25, 58G20.

Research partially supported by Danish Science Research Council Grant 9503564 . 


\section{CONTENTS}

Introduction 2

1. The Hörmander Index under Transversality Condition 4

2. The Maslov Index for Paths 5

3. The Fredholm Lagrangian Grassmannian 7

4. The Topology of the Fredholm Lagrangian Grassmannian 9

5. The Hörmander Index in Infinite Dimensions 15

6. An Example 17

7. General Boundary Data and Cauchy Data Spaces in Distribution Space $\quad 19$

8. Generalized Yoshida-Nicolaescu Formula in $H^{-\frac{1}{2}}(\Sigma) \quad 28$

Appendix A. A Characterization of Lagrangian Fredholm Pairs 35

$\begin{array}{ll}\text { References } & 38\end{array}$

\section{INTRODUCTION}

Our first purpose is to explain the topology of the Fredholm Lagrangian Grassmannian (Theorem 4.2). There have been various other treatments of this subject before (see, in chronological order, Swanson [18], Bojarski [1], Booss and Wojciechowski [3], Douglas and Wojciechowski [8], and Nicolaescu [12]). We want to unify the approaches and to clarify the underlying conditions from the very beginning. To us, the basic underlying topological fact is that the space of bounded self-adjoint Fredholm operators appears for the sums of the orthogonal projections of Fredholm pairs (our Appendix, Proposition A.2). Our approach is not based on delicate topological considerations like the Palais Theorem, [13]. Instead of that we discuss the topology more concretely by establishing in Section 4 a fibre bundle structure of a suitable subspace of the Fredholm Lagrangian Grassmannian with explicit local trivialization.

A second purpose is to clarify the notion of the Hörmander index. In Section 5 (inspired by Proposition 2.1), we have chosen to characterize the Hörmander index from the homotopy point of view, based on the notion of the Maslov index for paths. That interpretation yields also the slight generalization necessary for establishing the Hörmander index in infinite dimensions. To give an example, in Section 6, we recall from [2] an abstract, purely functional analytical spectral flow formula which identifies the spectral flow with the Maslov index in a quite general 
frame. In Theorem 6.4, we obtain a formula, similar to the AgranovičDynin type formulas in index theory, which describes the change of the spectral flow when replacing one domain by another one in terms of a Hörmander index.

Our third purpose is to apply our abstract functional analytical constructions and theorems to the concrete situation of a continuous family $\left\{A+C_{t}\right\}$ of symmetric elliptic differential operators of first order with fixed principal symbol over a compact connected smooth Riemannian manifold $M$ with boundary $\Sigma$. In Section 7 , we embed the space of abstract boundary data into the distribution space $H^{-\frac{1}{2}}(\Sigma)$ and study the $L_{2}$-extensions of the operator family which are defined by 'general elliptic boundary conditions' (i.e. extensions with compact resolvent). Some special features are explained for the case that the operator can be written in product form near the boundary.

In Section 8, we apply our abstract spectral flow formula and obtain a very general Yoshida-Nicolaescu type spectral flow formula over a manifold with boundary and, as special cases, related formulas over closed manifolds with fixed hypersurface. As compared to the famous papers by Yoshida [19] (dimension 3) and Nicolaescu [12] (general odd dimension, see also Cappell, Lee, and Miller [7]), the application of our strictly functional analytical approach yields not only a more direct and transparent proof of the formulas but also a series of generalizations. We need only the (weak) unique continuation property and not that the operators are of Dirac type. The hypersurface must have an orientable normal bundle, but need not separate the manifold. Neither do we assume the product form of the operators close to the hypersurface, and we can drop a variety of technical assumptions like the differentiability of the perturbation curve $\left\{C_{t}\right\}$; the invertibility of the operators at the endpoints 0,1 ; and the multiplicity one of all 0-eigenvalues.

We shall emphasize that our formula is written in the distribution space $H^{-\frac{1}{2}}(\Sigma)$, where the Cauchy data spaces and the traces of the maximal domain naturally belong. By finite-dimensional symplectic reduction we obtain, however, a version of the spectral flow formula which solely involves function spaces on the hypersurface.

Acknowledgments. We would like to thank M. de Gosson (Karlskrona), N. Otsuki (Tokyo) and K.P. Wojciechowski (Indianapolis) for stimulating questions and critical remarks. 


\section{The Hörmander Index under Transversality Condition}

We recall the definition of the intersection index of four Lagrangian subspaces in finite dimensions given in [11] by $L$. Hörmander. For natural $n$, we consider the Euclidean space $\mathbf{R}^{2 n}$ with the standard symplectic form $\omega\left((x, y),\left(x^{\prime}, y^{\prime}\right)\right):=\left(x, y^{\prime}\right)-\left(y, x^{\prime}\right)$ and the corresponding almost complex structure $J: \mathbf{R}^{2 n} \rightarrow \mathbf{R}^{2 n}$ with $J^{2}=-I$ dd and ${ }^{t} J=-J$. Clearly, $J$ yields an identification of $\mathbf{R}^{2 n}$ with $\mathbf{C}^{n}$. Let $\operatorname{Lag}(n)$ denote the set of Lagrangian subspaces of $\mathbf{R}^{2 n}$. It can be identified in a natural way with $U(n) / O(n)$ with fundamental group $\pi_{1}(U(n) / O(n)) \cong \mathbf{Z}$.

We choose $\lambda_{1}, \lambda_{2}, \mu_{1}, \mu_{2} \in \operatorname{Lag}(n)$ such that

$$
\lambda_{i} \cap \mu_{j}=\{0\} \quad \text { for } i, j=1,2 .
$$

Since the symplectic group acts transitively on the space of transversal pairs of Lagrangian subspaces, we can assume that $\lambda_{1}$ and $\mu_{2}$ are orthogonal. So, $J \lambda_{1}=\lambda_{1}^{\perp}=\mu_{2}$. We represent $\lambda_{2}$ as the graph of a linear map $A: \mu_{2} \rightarrow \lambda_{1}$ and $\mu_{1}$ as the graph of a linear map $B: \lambda_{1} \rightarrow \mu_{2}$ with $A \circ J$ and $J \circ B$ symmetric on $\lambda_{1}$. Then the Hörmander index is defined by

$$
\sigma_{\text {Hör }}\left(\lambda_{1}, \lambda_{2} ; \mu_{1}, \mu_{2}\right):=-\frac{1}{2} \operatorname{sign}\left(\begin{array}{cc}
-A \circ J & \text { Id } \\
\text { Id } & -J \circ B
\end{array}\right) .
$$

As it stands, the definition does not generalize to infinite dimensions without regularization. Fortunately, already in Hörmander [11] an alternative description of the Hörmander index was provided by using the Maslov cycle and the Maslov index. The Maslov cycle $\mathcal{M}_{\lambda}$ defined by $\lambda \in \operatorname{Lag}(n)$ is the set of Lagrangian subspaces given by

$$
\mathcal{M}_{\lambda}:=\{\theta \in \operatorname{Lag}(n) \mid \theta \cap \lambda \neq\{0\}\} .
$$

In general, different $\lambda$ will yield different Maslov cycles as sets. However, if we consider the Maslov cycle as homology class in the integer homology in codimension 1 , it gives the same class, namely the generator of

Note that the set

$$
H_{\frac{n(n+1)}{2}-1}(\operatorname{Lag}(n) ; \mathbf{Z}) \cong \mathbf{Z}
$$

$$
\operatorname{Lag}(n) \backslash \mathcal{M}_{\lambda}=\{\mu \in \operatorname{Lag}(n) \mid \mu \text { and } \lambda \text { transversal }\}
$$

is open, pathwise connected and, in fact, contractible because it can be identified with the space $\left\{A: \lambda \rightarrow \lambda \mid A={ }^{t} A\right\}$. Finally, we recall that the Maslov index mas $\left(\{C(t)\}_{t \in I}\right)$ for loops in $\operatorname{Lag}(n)$ is well defined as the intersection number with the Maslov cycle. It is independent of the specific choice of the Maslov cycle and depends solely on the homology class $[C]$ of the loop. 
Then, let $C_{1}: I=[0,1] \rightarrow \operatorname{Lag}(n) \backslash \mathcal{M}_{\mu_{1}}$ be a continuous curve connecting $C_{1}(0)=\lambda_{1}$ with $C_{1}(1)=\lambda_{2}$ and $C_{2}: I \rightarrow \operatorname{Lag}(n) \backslash \mathcal{M}_{\mu_{2}}$ a corresponding curve connecting $\lambda_{2}$ with $\lambda_{1}$. Then

Proposition 1.1. (Hörmander [11], Section 3.3) Under the transversality condition (1.1) and with the preceding construction of the curves $C_{1}, C_{2}$ we have

$$
\sigma_{\text {Hör }}\left(\lambda_{1}, \lambda_{2} ; \mu_{1}, \mu_{2}\right)=\operatorname{mas}\left(\left[C_{1}\right]+\left[C_{2}\right]\right) .
$$

Note. In [11], the transversality condition (1.1) arises naturally when describing the transition functions of the Maslov line bundle of a Lagrangian submanifold in the cotangent bundle. Another reason for that restriction may have been that at that time there did not exist a definition of the Maslov index for paths. In the next section we shall show how to get rid of the transversality condition. I.e., we establish the Hörmander index for arbitrary quadruples of Lagrangian subspaces in finite dimensions and prepare the infinite-dimensional case. To this end we recall the definition of the Maslov index for paths following our presentation in [2].

\section{The Maslov INDEX For Paths}

The Maslov (intersection) index for paths was introduced in [17] by J. Robbin and D. Salamon (see also the earlier definition of the Leray intersection index in the universal covering of $\operatorname{Lag}(n)$ given by $M$. de Gosson in [9] and the comprehensive study [6] by S. Cappell, R. Lee, and E. Miller). In [17], the Maslov index for paths was defined under somewhat restrictive conditions, namely only for smooth curves which have regular crossings with the Maslov cycle. The definition was re-written and generalized in [2] by the present authors. The new approach was inspired by J. Phillips, [14]. We shall summarize that functional analytical definition.

Let $\mathcal{H}$ be a real symplectic separable Hilbert space. We denote the inner product by $(\cdot, \cdot)$, the symplectic form by $\omega(\cdot, \cdot)$, and the corresponding almost complex structure by $J$, so that $\omega(x, y)=(J x, y)$, $J^{2}=-1$, and ${ }^{t} J=-J$.

Let $\operatorname{Lag}(\mathcal{H})$ denote the space of all Lagrangian subspaces with the topology defined by the operator norm of the projections. If $\operatorname{dim} \mathcal{H}=$ $+\infty$, it is contractible by Kuiper's Theorem. For fixed $\lambda \in \operatorname{Lag}(\mathcal{H})$ 
we notice that $J \lambda=\lambda^{\perp}$. So, any $\mu \in \operatorname{Lag}(\mathcal{H})$ can be obtained as the image of $\lambda^{\perp}$ under a suitable unitary transformation

$$
\mu=U\left(\lambda^{\perp}\right) .
$$

Here we consider the real symplectic Hilbert space $\mathcal{H}$ as a complex Hilbert space by the almost complex structure. Note that $U$ is not uniquely determined by $\mu$. Actually, from $\mathcal{H} \cong \lambda \otimes \mathbf{C}$ we obtain a complex conjugation so that we can define the complex transpose

$$
T_{U}:=\overline{U^{*}}
$$

and a unitary operator $W:=U^{T} U$ which can be defined invariantly as the complex generator of the Lagrangian space $\mu$ relative to $\lambda$. We have

$$
\text { Id }+W \text { is a Fredholm operator } \Longleftrightarrow(\mu, \lambda) \text { is a Fredholm pair. }
$$

In particular, we have

$$
\operatorname{ker}(\operatorname{Id}+W)=(\mu \cap \lambda) \otimes \mathbf{C}=(\mu \cap \lambda)+J(\mu \cap \lambda) .
$$

For the definition of the space of Fredholm pairs and the characterization of Lagrangian Fredholm pairs we refer to the Appendix.

We define the Maslov (intersection) index mas $\left(\left\{\mu_{t}\right\}, \lambda\right)$ of the fixed Lagrangian subspace $\lambda$ with a curve $\left\{\mu_{t}\right\}$ of Lagrangian subspaces: Because of (2.1) we shall assume that the curve $\left\{\mu_{t}\right\}$ stays in the Fredholm Lagrangian Grassmannian defined by

$$
\mathcal{F L}_{\lambda}:=\{\mu \in \operatorname{Lag}(\mathcal{H}) \mid(\lambda, \mu) \text { Fredholm pair in } \mathcal{H}\} \text {. }
$$

Then, in an analoguous way to [14], we count the change of the eigenvalues near -1 little by little. For example, between $t=0$ and $t=t^{\prime}$ we plot the spectrum of the complex generator $W_{t}$ close to $e^{i \pi}$. In general, there will be no parametrization available of the spectrum near -1 . For sufficiently small $t^{\prime}$, however, we can find barriers $e^{i(\pi+\theta)}$ and $e^{i(\pi-\theta)}$ such that no eigenvalues get lost through the barriers on the interval $\left[0, t^{\prime}\right]$. Then we count the number of eigenvalues (with multiplicity) of $W_{t}$ between $e^{i \pi}$ and $e^{i(\pi+\theta)}$ at the right and the left end of the interval $\left[0, t^{\prime}\right]$ and subtract. Repeating that procedure over the length of the whole $t$-interval $[0,1]$ gives the Maslov intersection index mas $\left(\left\{\mu_{t}\right\}, \lambda\right)$ without any assumptions about smoothness of the curve or 'normal crossings' in the sense of [17].

By (2.2), the Maslov index can be considered as the intersection number between the given curve $\left\{\mu_{t}\right\}$ and the Maslov cycle

$$
\mathcal{M}_{\lambda}:=\left\{\theta \in \mathcal{F} \mathcal{L}_{\lambda} \mid \theta \cap \lambda \neq\{0\}\right\}
$$


Note that the Maslov index for paths depends essentially on the choice of the reference Maslov cycle. This dependence is expressed by the Hörmander index. We rewrite Proposition 1.1 in the following way:

Let $C: I \rightarrow \operatorname{Lag}(n)$ be an arbitrary continuous curve connecting $\lambda_{1}$ with $\lambda_{2}$ and let the curve $C_{1}$ connect $\lambda_{1}$ with $\lambda_{2}$ in $\operatorname{Lag}(n) \backslash \mathcal{M}_{\mu_{1}}$ and the curve $C_{2}$ connect $\lambda_{2}$ with $\lambda_{1}$ in $\operatorname{Lag}(n) \backslash \mathcal{M}_{\mu_{2}}$ as in Proposition 1.1. By construction, we have $\operatorname{mas}\left(C_{j}, \mu_{j}\right)=0$ for $j=1,2$. Let us denote the catenation of two paths by $*$. Then

$$
\begin{aligned}
\operatorname{mas}\left(C_{2} * C_{1}, \mu_{1}\right) & =\operatorname{mas}\left(C_{2} * C *(-C) * C_{1}, \mu_{1}\right) \\
& =\operatorname{mas}\left((-C) * C_{1}, \mu_{1}\right)+\operatorname{mas}\left(C_{2} * C, \mu_{1}\right) \\
& =\operatorname{mas}\left((-C) * C_{1}, \mu_{1}\right)+\operatorname{mas}\left(C_{2} * C, \mu_{2}\right) \\
& =-\operatorname{mas}\left(C, \mu_{1}\right)+\operatorname{mas}\left(C, \mu_{2}\right),
\end{aligned}
$$

where the last two integers are the Maslov indices of paths. Here, we exploited the additivity of the Maslov index for paths under catenation of the paths and the invariance of the Maslov index for.cycles under change of the Maslov cycle.

The preceding argument gives us the following generalization of the Hörmander index for arbitrary quadruples of Lagrangian subspaces in finite dimensions:

Proposition 2.1. Let $C: I \rightarrow \operatorname{Lag}(n)$ be an arbitrary continuous curve connecting $\lambda_{1}$ with $\lambda_{2}$. Then the integer

$$
\sigma_{\text {Hör }}\left(\lambda_{1}, \lambda_{2} ; \mu_{1}, \mu_{2}\right):=\operatorname{mas}\left(\{C(t)\}, \mu_{2}\right)-\operatorname{mas}\left(\{C(t)\}, \mu_{1}\right)
$$

is well defined and does not depend on the choice of the curve $\{C(t)\}$ joining $\lambda_{1}$ to $\lambda_{2}$.

Proposition 2.1 suggests a similar definition of the Hörmander index in infinite dimensions (see below Definition 5.2).

\section{The Fredholm Lagrangian Grassmannian}

We shall investigate how the Fredholm Lagrangian Grassmannian depends on the reference space $\lambda$. If $\operatorname{dim} \mathcal{H}=2 n<+\infty$, we have $\mathcal{F} \mathcal{L}_{\lambda}=\operatorname{Lag}(n)$. In infinite dimensions, $\mathcal{F} \mathcal{L}_{\lambda}$ is a true subset of $\operatorname{Lag}(\mathcal{H})$. For instance, it does not contain $\lambda$, but $\lambda$ can be approximated by a sequence in $\mathcal{F} \mathcal{L}_{\lambda}$. For instance, let $A: \lambda \stackrel{\cong}{\longrightarrow} \lambda^{\perp}=J(\lambda)$ such that $J \circ A: \lambda \rightarrow \lambda$ is symmetric. Then for all $\varepsilon>0$,

$$
\mu_{\varepsilon}:=\operatorname{graph}(\varepsilon A)=\{x+\varepsilon A x \mid x \in \lambda\}
$$

is a Lagrangian subspace of $\mathcal{H}$, and we have

$$
\mu_{\varepsilon} \cap \lambda=\{0\} \text { and } \mu_{\varepsilon}+\lambda=\mathcal{H} \text {. }
$$


Clearly, the convergence $\mu_{\varepsilon} \rightarrow \lambda$ is understood in the sense that the orthogonal projections $\pi_{\mu_{\varepsilon}}$ of $\mathcal{H}$ onto $\mu_{\varepsilon}$ converge to the projection $\pi_{\lambda}$. Consequently we have

$$
\lambda \in \overline{\mathcal{F} \mathcal{L}_{\lambda}} \backslash \mathcal{F} \mathcal{L}_{\lambda} .
$$

A partial answer to the problem of $\lambda$-dependence of $\mathcal{F} \mathcal{L}_{\lambda}$ is provided by the following proposition.

Proposition 3.1. (a) On the space $\operatorname{Lag}(\mathcal{H})$ an equivalence relation is defined by

$$
\lambda \sim \mu: \Longleftrightarrow \operatorname{dim} \lambda /(\lambda \cap \mu)<+\infty \quad \text { for } \lambda, \mu \in \operatorname{Lag}(\mathcal{H}) .
$$

(b) If $\lambda \sim \mu$, we have $\mathcal{F} \mathcal{L}_{\lambda}=\mathcal{F} \mathcal{L}_{\mu}$.

The proof of the preceding proposition is an easy consequence of the following more general observation:

Lemma 3.2. Let $\lambda \in \operatorname{Lag}(\mathcal{H})$ and let $W \subset \lambda$ be a closed subspace of finite codimension in $\lambda$. Then we have

$$
(\lambda, \mu) \in \operatorname{Fred}^{2}(\mathcal{H}) \Longleftrightarrow(W, \mu) \in \operatorname{Fred}^{2}(\mathcal{H})
$$

for any $\mu \in \operatorname{Lag}(\mathcal{H})$.

Remark 3.3. Clearly, from the assumptions about $W$ we have that $W$ is contained in its annihilator $W^{0}$, that the factor space $W^{0} / W$ is naturally a symplectic vector space and $\operatorname{dim} W^{0} / W<+\infty$.

Proof. The lemma asserts that

$$
\mathcal{F} \mathcal{L}_{W}:=\left\{\mu \in \operatorname{Lag}(\mathcal{H}) \mid(W, \mu) \in \operatorname{Fred}^{2}(\mathcal{H})\right\}=\mathcal{F} \mathcal{L}_{\lambda} .
$$

Let $\mu \in \mathcal{F} \mathcal{L}_{\lambda}$. We have $\operatorname{dim} \lambda \cap \mu \leq \operatorname{dim} W \cap \mu+\operatorname{dim} \lambda / W<+\infty$. Moreover, $W+\mu \subset \lambda+\mu \subset \mathcal{H}$. So, $\lambda+\mu$ is an intermediate space between the closed subspace $W+\mu$ of finite codimension and the whole space $\mathcal{H}$. So, $\lambda+\mu$ is closed and $\operatorname{dim} \mathcal{H} /(\lambda+\mu)<+\infty$.

We prove the opposite inclusion. Clearly,

$$
\operatorname{dim} W \cap \mu \leq \operatorname{dim} \lambda \cap \mu<+\infty .
$$

To prove that $W+\mu$ is closed in $\mathcal{H}$, we consider the short exact sequence

$$
0 \longrightarrow \lambda \cap \mu \stackrel{j}{\longrightarrow} \lambda \oplus \mu \stackrel{\tau}{\longrightarrow} \lambda+\mu \longrightarrow 0,
$$

where $j(a):=a \oplus-a \in \mathcal{H} \times \mathcal{H}$ and $\tau(a \oplus b):=a+b$. We have

$$
\tau^{-1}(W+\mu)=W \oplus \mu+j(\lambda \cap \mu) .
$$

Here $W \oplus \mu$ is closed in $\lambda \oplus \mu$ and $j(\lambda \cap \mu)$ is finite-dimensional. It follows that $W+\mu$ is closed and of finite codimension. 
Now the proof of Proposition 3.1 follows at once.

Proof of Proposition 3.1. (a) We prove only the symmetry. Consider the following diagram

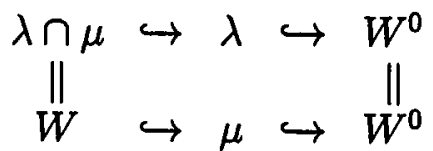

where $W:=\lambda \cap \mu$. Then $\operatorname{dim}\left(W^{0} / W\right)=2 k<+\infty$ if $\operatorname{dim} \lambda /(\lambda \cap \mu)=$ $k<+\infty$. Then also $\operatorname{dim} \mu / W=k$. Similarly we prove the transitivity.

(b) We take $W:=\lambda \cap \mu$.

\section{The Topology of the Fredholm Lagrangian GRASSMANNIAN}

Our next goal is to give an elementary proof of the well-known fact that the fundamental group of $\mathcal{F} \mathcal{L}_{\lambda}$ is infinite cyclic. This result has been obtained first by R. Swanson in [18], Theorem 2.1 and Corollary, for the smaller Grassmannian which is obtained by inductive limit from the Lagrangians in finite dimension. His proof exploits Palais' Theorem on the topology of infinite-dimensional spaces (see [13]). A different approach was chosen in Douglas and Wojciechowski [8] (see also Booss and Wojciechowski [3] and [4] for the non-Lagrangian case) for a Grassmannian which consists of all $\mu \in \mathcal{F} \mathcal{L}_{\lambda}$ such that $\pi_{\mu}+\pi_{\lambda}$ differ from identity by a compact operator. This Grassmannian arises naturally when one considers self-adjoint boundary conditions for first order elliptic differential operators which are defined by pseudo-differential projections with the same principal symbol as the Atiyah-PatodiSinger spectral projection. The proof there exploits the homogeneous properties of the corresponding reduced groups and quotients.

It follows from Palais' Theorem, that all the mentioned spaces are homotopy equivalent. Nevertheless, we find it worthwhile to provide an explicit and elementary proof for the full Lagrangian Grassmannian $\mathcal{F} \mathcal{L}_{\lambda}$.

We shall rely on a couple of elementary properties of Fredholm pairs of Lagrangian subspaces in symplectic Hilbert space established below in Appendix A.

Definition 4.1. Let $\lambda$ be a Lagrangian subspace of a symplectic Hilbert space $\mathcal{H}$. (a) We shall use the notation $\mathcal{C}_{\prec \lambda}^{\text {fin }}$ for the set of closed subspaces of $\lambda$ of finite codimension. 
(b) Let $W \in \mathcal{C}_{\alpha \lambda}^{\text {fin }}$. We shall use the notation $\mathcal{L}_{\succ W}$ for the set of Lagrangian subspaces of $\mathcal{H}$ which contain $W$.

We shall prove

Theorem 4.2. Let $\lambda$ be a Lagrangian subspace of a symplectic Hilbert space $\mathcal{H}$.

(a) The inclusions

$$
\mathcal{F} \mathcal{L}_{W}^{(0)}:=\left\{\theta \in \mathcal{F} \mathcal{L}_{\lambda} \mid \theta \cap W=\{0\}\right\} \hookrightarrow \mathcal{F} \mathcal{L}_{\lambda} \quad \text { for } W \in \mathcal{C}_{\prec \lambda}^{\text {fin }}
$$

define an isomorphism

$$
\underset{W \rightarrow\{0\}}{\operatorname{ind}-\lim } \pi_{1}\left(\mathcal{F} \mathcal{L}_{W}^{(0)}\right) \quad \stackrel{\cong}{\longrightarrow} \quad \pi_{1}\left(\mathcal{F} \mathcal{L}_{\lambda}\right) .
$$

(b) There is a natural isomorphism

$$
\pi_{1}\left(\mathcal{F L}_{W}^{(0)}\right) \stackrel{\cong}{\longrightarrow} \pi_{1}\left(\operatorname{Lag}\left(W^{0} / W\right)\right) \cong \mathbf{Z}
$$

for each $W \in \mathcal{C}_{\prec \lambda}^{\text {fin }}$.

By combining (a) and (b) we obtain

Corollary 4.3. The Fredholm Lagrangian Grassmannian $\mathcal{F} \mathcal{L}_{\lambda}$ has the fundamental group

$$
\pi_{1}\left(\mathcal{F} \mathcal{L}_{\lambda}\right) \cong \mathbf{Z}
$$

for any Lagrangian subspace $\lambda$ of $\mathcal{H}$.

The proof of Theorem 4.2 will follow from two propositions which are of independent interest. First we shall show that any path in $\mathcal{F} \mathcal{L}_{\lambda}$ is transversal for a suitable choice of a closed subspace $W \subset \lambda$ of finite codimension. More generally, we have

Proposition 4.4. Let $K \subset \mathcal{F} \mathcal{L}_{\lambda}$ be compact. Then there exists a $W \in \mathcal{C}_{\{\lambda}^{\text {fin }}$ such that $\mu \cap W=\{0\}$ for all $\mu \in K$.

Proof. Let $\mu_{0} \in K$. Then the sum of the orthogonal projections $\pi_{\lambda}+\pi_{\mu_{0}}$ is a Fredholm operator by Proposition A.2 of the Appendix and we have

$$
\operatorname{ker}\left(\pi_{\lambda}+\pi_{\mu_{0}}\right)=J\left(\lambda \cap \mu_{0}\right)
$$

by (A.2). Let

$$
h:=\left(J\left(\lambda \cap \mu_{0}\right)\right)^{\perp}=\lambda+\left(\lambda^{\perp} \cap\left(J\left(\lambda \cap \mu_{0}\right)\right)^{\perp}\right) .
$$

Then the operator $\pi_{\lambda}+\pi_{\mu_{0}}$ is injective on $h$ and its range $\lambda+\mu_{0}$ is closed. Hence there exists an open neighbourhood $U$ of $\mu_{0}$ in $\mathcal{F} \mathcal{L}_{\lambda}$ such that $\pi_{\lambda}+\pi_{\mu}$ is injective on $h$ for all $\mu \in K \cap U$. Since $K$ compact, 
a finite set $U_{1}, \ldots, U_{N}$ of such neighbourhoods covers the whole of $K$. Then

$$
W:=\bigcap_{j=1}^{N}\left(\left(\lambda \cap \mu_{j}\right)^{\perp} \cap \lambda\right)
$$

satisfies our requirement for suitable choices of $\mu_{j} \in U_{j} \cap K$.

The main technical result of this section is the following one.

Proposition 4.5. Let $W$ be a closed subspace of finite codimension of a Lagrangian subspace $\lambda$ in a symplectic Hilbert space $\mathcal{H}$. Then the well-known mapping

defines a fibre bundle.

$$
\begin{aligned}
& \rho_{W}: \mathcal{F L}_{W}^{(0)} \rightarrow \quad \operatorname{Lag}\left(W^{0} / W\right) \\
& \mu \quad \mapsto \quad\left(\left(\mu \cap W^{0}\right)+W\right) / W
\end{aligned}
$$

We shall prepare the proof of the proposition by introducing systems of open neighbourhoods in the total space and the basis.

Lemma 4.6. (a) Let $\mathcal{H}, \lambda, W$ as before and let $\theta \in \mathcal{L}_{\succ W}$ '(see Definition 4.1). Then

$$
U_{\theta}:=\left\{\mu \in \mathcal{F} \mathcal{L}_{W}^{(0)} \mid \mu \cap \theta=\{0\}\right\}
$$

is an open subset of the total space $\mathcal{F} \mathcal{L}_{W}^{(0)}$ and we have

$$
\bigcup\left\{U_{\theta} \mid \theta \in \mathcal{L}_{\succ W}\right\}=\mathcal{F} \mathcal{L}_{W}^{(0)} .
$$

(b) Let $\bar{\theta}:=\theta / W \in \operatorname{Lag}\left(W^{0} / W\right)$. Then the set

$$
U_{\bar{\theta}}:=\left\{L \in \operatorname{Lag}\left(W^{0} / W\right) \mid L \cap \bar{\theta}=\{0\}\right\}
$$

is an open subset of the basis $\operatorname{Lag}\left(W^{0} / W\right)$ and the union of all such subsets covers the basis.

Proof. To see that $U_{\theta}$ is open, we apply Proposition A.2 of the Appendix A. So, $\pi_{\theta}+\pi_{\mu}$ is an isomorphism. Then we have an open neighbourhood of $\mu$ so that each element in the neighbourhood is invertible.

For given $\mu \in \mathcal{F} \mathcal{L}_{W}^{(0)}$ one finds easily a $\theta \in \operatorname{Lag}(\mathcal{H})$ with $\theta \supset W$ and $\theta \cap \mu=\{0\}$. That gives the claimed open covering.

In the same way, (b) follows.

Lemma 4.7. The mapping

is surjective.

$$
\rho_{W}: U_{\theta} \longrightarrow U_{\bar{\theta}}
$$


Proof. First we prove that $\rho_{W}\left(U_{\theta}\right) \subset U_{\bar{\theta}}$. Take $\mu \in U_{\theta}$. Then $\mu \cap \theta=$ $\{0\}$ and $\mu \cap W=\{0\}$. We shall show that each

$$
z \in\left(\left(\mu \cap W^{0}\right)+W\right) / W \cap \theta / W
$$

vanishes. By (4.1), the class $z$ can be written as

$$
z=[x+w]=[y]
$$

where $x \in \mu \cap W^{0}, w \in W, y \in \theta$, and [.] denotes the class modulo $W$. Then

$$
\mu \ni x=y+w^{\prime} \in \theta \text { for suitable } w^{\prime} \in W \subset \theta .
$$

So, $x=0$. That implies $y \in W$, so $z=0$ as class modulo $W$.

Next we prove $\rho_{W}\left(U_{\theta}\right)=U_{\bar{\theta}}$, that is the surjectivity of $\rho_{W}: U_{\theta} \rightarrow$ $U_{\bar{\theta}}$. We decompose $\mathcal{H}$ into two, respectively four mutually orthogonal subspaces

$$
\begin{aligned}
\mathcal{H} & =\quad \begin{array}{c}
\theta \\
=
\end{array} W^{\perp} \cap \theta+W\left(W^{\perp} \cap \theta\right)+J(W)
\end{aligned}
$$

It follows

$$
W^{0}=(J W)^{\perp}=W^{\perp} \cap \theta+W+J\left(W^{\perp} \cap \theta\right) .
$$

From (4.2) we see that $\mathcal{H}$ is the product of two symplectic subspaces, namely the (infinite-dimensional, if $\mathcal{H}$ is infinite-dimensional) symplectic subspace $W+J W$ with, e.g., the Lagrangian subspaces $W$ and $J W$ and the finite-dimensional symplectic subspace $W^{\perp} \cap \theta+J\left(W^{\perp} \cap \theta\right)$ with, e.g., the Lagrangian subspaces $W^{\perp} \cap \theta$ and $J\left(W^{\perp} \cap \theta\right)$. Note that the smaller symplectic subspace of $\mathcal{H}$ is naturally isomorphic with the factor space $W^{0} / W$.

Then, let $L \in \operatorname{Lag}\left(W^{0} / W\right)$. It can be identified with a subspace $L \subset W^{\perp} \cap \theta+J\left(W^{\perp} \cap \theta\right)$. Then $\mu_{L}:=L+J W$ is a Lagrangian subspace of $\mathcal{H}$ and we have $\rho_{W}\left(\mu_{L}\right)=L$.

We shall exploit the decomposition (4.2) of $\mathcal{H}$ into four mutually orthogonal subspaces a little further. We prove the following lemma.

Lemma 4.8. Let $\lambda, W, \theta$ as above. Let $\mu \in U_{\theta}$. Then there exist linear mappings

$$
\begin{aligned}
& \alpha: J\left(W^{\perp} \cap \theta\right) \longrightarrow W^{\perp} \cap \theta \\
& \gamma: J\left(W^{\perp} \cap \theta\right) \longrightarrow W
\end{aligned}
$$

such that each $z \in \mu \cap W^{0}$ can be written in the form

$$
z=x+\alpha x+\gamma x \quad \text { with } x \in J\left(W^{\perp} \cap \theta\right) .
$$


Proof. Since $\mu$ intersects $\theta$ transversally, there is a map $A: J \theta \rightarrow \theta$ such that $A \circ J$ self-adjoint on $\theta$ and $\mu=\{u+A u \mid u \in J \theta\}$. We decompose $u=x+y$ with $x \in J\left(W^{\perp} \cap \theta\right)$ and $y \in J(W)$ according to the decomposition of $J \theta$ in (4.2). With regard to this decomposition, the mapping $A$ can be written as a two-by-two matrix $\left(\begin{array}{ll}\alpha & \beta \\ \gamma & \delta\end{array}\right)$. More explicitly, we have

$$
A u=\alpha x+\beta y+\gamma x+\delta y,
$$

where

$$
\begin{aligned}
& \alpha: J\left(W^{\perp} \cap \theta\right) \longrightarrow W^{\perp} \cap \theta \\
& \beta: J(W) \longrightarrow W^{\perp} \cap \theta \\
& \gamma: J\left(W^{\perp} \cap \theta\right) \longrightarrow W \\
& \delta: J(W) \longrightarrow W
\end{aligned}
$$

We notice that

$$
\alpha \circ J, \delta \circ J \text { self-adjoint, and }{ }^{t}(\beta \circ J)=\gamma \circ J .
$$

Now, let $z \in \mu \cap W^{0}$. It can be written as

$$
z=u+A u=x+y+\alpha x+\beta y+\gamma x+\delta y .
$$

From the decomposition (4.2) it follows that the component $y$ in $J(W)$ must vanish. So,

$$
z=x+\alpha x+\gamma x
$$

Corollary 4.9. Let $\lambda, W, \theta$ as above. Let $\mu=\{u+A u \mid u \in J \theta\} \in U_{\theta}$ with $A=\left(\begin{array}{ll}\alpha & \beta \\ \gamma & \delta\end{array}\right)$ relative to the decompositions $J \theta=J\left(W^{\perp} \cap \theta\right)+$ $J(W)$ and $\theta=W^{\perp} \cap \theta+W$. As before, we identify $W^{0} / W$ with $W^{\perp} \cap \theta+J\left(W^{\perp} \cap \theta\right)$. Then

$$
\rho_{W}(\mu)=\left\{x+\alpha x \mid x \in J\left(W^{\perp} \cap \theta\right)\right\} .
$$

In particular, two $\mu, \mu^{\prime} \in U_{\theta}$ belong to the same fibre, i.e., $\rho_{W}(\mu)=$ $\rho_{W}\left(\mu^{\prime}\right)$ if and only if $\alpha=\alpha^{\prime}$.

We are ready to prove Proposition 4.5. 
Proof. We define a local trivialization by the following diagram:

$$
\begin{array}{ccc}
U_{\bar{\theta}} \times F & \stackrel{\tau}{\longrightarrow} & U_{\theta} \\
p \searrow & \downarrow \rho_{W} \\
& U_{\bar{\theta}}
\end{array}
$$

Here, $p$ denotes the projection onto the first component. We take

$$
F:=\mathcal{B}\left(J W, W^{\perp} \cap \theta\right)+\mathcal{B}_{\mathrm{sa}}(J W, W)
$$

where $\mathcal{B}\left(J W, W^{\perp} \cap \theta\right)$ denotes the vector space of bounded operators from $J W$ to $W^{\perp} \cap \theta$ and $\mathcal{B}_{\text {sa }}(J W, W)$ the vector space of bounded operators from $J W$ to $W$ which become self-adjoint on $W$ by combination with $J$. For a footpoint $L \in U_{\bar{\theta}}$ and a point in the fibre $(\beta, \delta) \in F$, we define

$$
\tau(L ; \beta, \delta):=\left\{u+A u \mid A=\left(\begin{array}{ll}
\alpha_{L} & \beta \\
\gamma_{\beta} & \delta
\end{array}\right), u \in J \theta\right\},
$$

with the decomposition $\left.J \theta=J\left(W^{\perp} \cap \theta\right)+J W\right\}$. The operator $\alpha_{L}$ : $J\left(W^{\perp} \cap \theta\right) \rightarrow W^{\perp} \cap \theta$ with $\alpha_{L} \circ J$ self-adjoint is uniquely determined by the condition

$$
L=\left\{x+\alpha_{L}(x) \mid x \in J\left(W^{\perp} \cap \theta\right)\right\}
$$

(see Corollary 4.9). As a consequence, we get $\tau$ surjective and injective. By definition of $\alpha_{L}$ from $L$ we get the commutativity of the diagram $(4.5)$.

Now we put the various pieces together.

Proof of Theorem 4.2. To get the inductive limit right, we consider two spaces $\widetilde{W}, W \in \mathcal{C}_{\langle\lambda}^{\text {fin }}$ with $\widetilde{W} \subset W$. So

$$
\mathcal{F} \mathcal{L}_{W}=\mathcal{F} \mathcal{L}_{\widetilde{W}}=\mathcal{F} \mathcal{L}_{\lambda}
$$

and

$$
\mathcal{F} \mathcal{L}_{W}^{(0)} \subset \mathcal{F} \mathcal{L}_{\bar{W}}^{(0)} \subset \mathcal{F} \mathcal{L}_{\lambda} .
$$

Recall that $\mathcal{L}_{\succ W}$ denotes the set of Lagrangian subspaces of $\mathcal{H}$ which contain $W$. Clearly we have an isomorphism

$$
\begin{array}{ccc}
\mathcal{L}_{\succ W} & \cong & \operatorname{Lag}\left(W^{0} / W\right) \\
\theta & \mapsto / W & \theta / W
\end{array}
$$


and a corresponding isomorphism for $\widetilde{W}$. Now let $C: I \rightarrow \mathcal{F} \mathcal{L}_{\lambda}$ be a curve which is transversal to $W$. So, it induces a curve $C^{\prime}: I \rightarrow \mathcal{F} \mathcal{L}_{W}^{(0)}$ Then we have the following commutative diagram

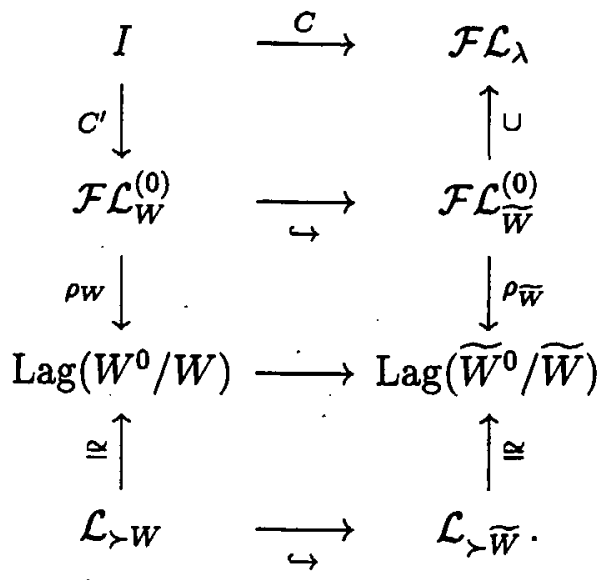

By (4.6), the mapping

$$
\underset{W \rightarrow\{0\}}{\operatorname{ind}-\lim } \pi_{1}\left(\mathcal{F} \mathcal{L}_{W}^{(0)}\right) \longrightarrow \pi_{1}\left(\mathcal{F} \mathcal{L}_{\lambda}\right)
$$

is well defined and injective. By Proposition 4.4, it is surjective. That proves (a).

To prove (b), we consider the exact homotopy sequence for the fibre bundle $\rho_{W}: \mathcal{F} \mathcal{L}_{W}^{(0)} \rightarrow \operatorname{Lag}\left(W^{0} / W\right)$

$$
\pi_{1}(F) \longrightarrow \pi_{1}\left(\mathcal{F} \mathcal{L}_{W}^{(0)}\right) \underset{\rho_{W}}{\longrightarrow} \pi_{1}\left(\operatorname{Lag}\left(W^{0} / W\right)\right) \longrightarrow \pi_{0}(F)
$$

and notice that $F$ is a vector space, hence contractible.

\section{The Hörmander INDEX in INFinite Dimensions}

Let $\mathcal{H}$ be a symplectic Hilbert space and $\lambda, \mu \in \operatorname{Lag}(\mathcal{H})$. We assume that $\lambda \sim \mu$, i.e., $\operatorname{dim} \lambda /(\lambda \cap \mu)<+\infty$. So $\mathcal{F} \mathcal{L}_{\lambda}=\mathcal{F} \mathcal{L}_{\mu}$. Let $C: I \rightarrow$ $\mathcal{F} \mathcal{L}_{\lambda}$ be a continuous curve. Then the difference

$$
\operatorname{mas}(C, \mu)-\operatorname{mas}(C, \lambda)
$$

depends solely on the endpoints of the curve $C$. Indeed, let $\widetilde{C}: I \rightarrow$ $\mathcal{F} \mathcal{L}_{\lambda}$ be a second curve connecting $C(0)$ and $C(1)$. We consider the closed path $-\widetilde{C} * C$. Recall that $*$ denotes catenation under suitable re-parametrization of the paths. For loops, the Maslov index does not depend on the choice of the Maslov cycle. That is an immediate consequence of Theorem 4.2 . 
More precisely, the central part

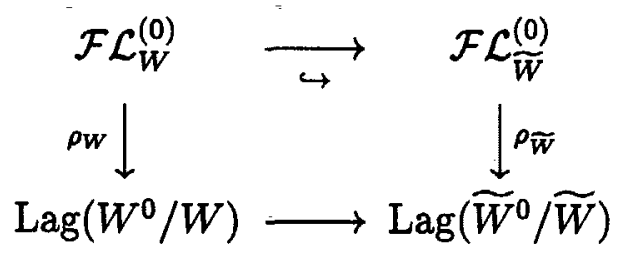

of the commutative diagram (4.6) yields a finite-dimensional symplectic reduction: it permits to express the Maslov index for loops in the Fredholm Lagrangian Grassmannian by the Maslov index of the corresponding loop of Lagrangian subspaces of a suitable symplectic vector space of finite dimension. But for finite dimension, it is well established that the Maslov index for loops is independent of the choice of the Maslov cycle.

Remark 5.1. The finite-dimensional symplectic reduction works also for paths. Let $C: I \rightarrow \mathcal{F} \mathcal{L}_{\lambda}$ and $W \in \mathcal{C}_{\prec \lambda}^{\text {fin }}$ such that range $C \subset \mathcal{F} \mathcal{L}_{W}^{(0)}$. So,

$$
\begin{aligned}
& \text { I } \\
& \downarrow C
\end{aligned}
$$

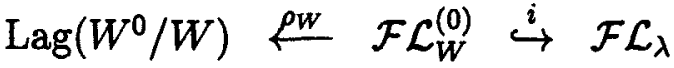

Then we have the following commutative diagram

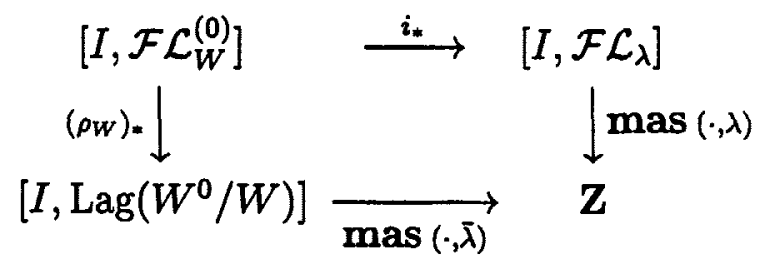

Here $[\cdot, \cdot]$ denotes the homotopy classes of mappings with fixed endpoints.

We return to our argument about the Maslov index of cycles. So $\operatorname{mas}(-\widetilde{C} * C, \lambda)=\operatorname{mas}(-\widetilde{C} * C, \mu)$. By additivity under catenation it follows that (5.1) is, as claimed, invariant under any change of $C$ which keeps the endpoints fixed. So:

Definition 5.2. The Hörmander index

$$
\sigma_{\text {Hör }}(C(0), C(1) ; \lambda, \mu):=\operatorname{mas}(C, \mu)-\operatorname{mas}(C, \lambda)
$$

is well defined.

The following elementary properties of the Hörmander index follow immediately from the definition. 
Proposition 5.3. The Hörmander index is skew-symmetric in its first two arguments and also in its last two arguments.

\section{AN EXAMPLE}

To give an example, we relate the spectral flow and the Hörmander index in the following way. We recall Krein's construction of the space of abstract boundary values for closed symmetric operators. That space carries a natural symplectic structure as pointed out in [2].

More precisely, let $\mathcal{H}$ be a real separable Hilbert space and $A$ a (unbounded) closed symmetric operator defined on the domain $D_{\min }$ which is supposed to be dense in $\mathcal{H}$. Let $A^{*}$ denote its adjoint operator with domain $D_{\max }$. Clearly, we have $\left.A^{*}\right|_{D_{\min }}=A$ and $A^{*}$ is the maximal closed extension of $A$ in $\mathcal{H}$. For a better understanding one may think of $A$ as an elliptic symmetric differential operator of first order on a compact smooth Riemannian manifold $X$ with boundary. (See also the following sections where a variety of concrete settings will be discussed).

For a given symmetric $A$ we want to discuss all self-adjoint extensions according to the von Neumann approach. To this end, we form the space $\boldsymbol{\beta}$ of abstract boundary values with the abstract trace map $\gamma$ in the following way:

$$
\begin{array}{ccc}
D_{\max } & \stackrel{\gamma}{\rightarrow} & D_{\max } / D_{\min }=: \boldsymbol{\beta} \\
x & \mapsto & \gamma(x)=[x]:=x+D_{\min }
\end{array}
$$

The space $\boldsymbol{\beta}$ becomes a symplectic Hilbert space with the scalar product induced by the graph norm

$$
(x, y)_{\mathcal{G}}:=(x, y)+\left(A^{*} x, A^{*} y\right)
$$

and the symplectic form given by Green's form

$$
\omega([x],[y]):=\left(A^{*} x, y\right)-\left(x, A^{*} y\right) \quad \text { for }[x],[y] \in \beta \text {. }
$$

For $D_{\min } \subset D \subset D_{\max }$ we set

$$
A_{D}:=\left.A^{*}\right|_{D}
$$

and get

$$
\begin{aligned}
A_{D} \text { closed } & \Longleftrightarrow \gamma(D) \text { closed } \\
A_{D} \text { self-adjoint } & \Longleftrightarrow \gamma(D) \text { Lagrangian }
\end{aligned}
$$

$A_{D}$ has compact resolvent $\Longleftrightarrow \quad D \hookrightarrow \mathcal{H}$ compact in graph norm.

Definition 6.1. We define the abstract Cauchy data space as subspace of $\boldsymbol{\beta}$ by

$$
\gamma(S) \quad \text { with } S:=\operatorname{ker} A^{*}
$$


We make two assumptions.

Assumption 6.2. First, we assume that $A$ admits at least one selfadjoint Fredholm extension $A_{D}$. Actually, we shall assume a little more, namely that $A$ has a self-adjoint extension $A_{D}$ with compact resolvent.

Then it follows (see [2], Proposition 3.5) that $\gamma(S)$ is a (closed) Lagrangian subspace of $\boldsymbol{\beta}$ and $(\gamma(S), \gamma(D))$ is a Fredholm pair of subspaces of $\boldsymbol{\beta}$. In particular we have $\gamma(S) \in \mathcal{F} \mathcal{L}_{\gamma(D)}$.

Assumption 6.3. Second and additionally, we assume that we are given a continuous curve $\left\{C_{t}\right\}$ in the space of bounded self-adjoint operators on $\mathcal{H}$ and that the operators $A^{*}+C_{t}-s$ for small $s$ have no 'inner solutions', i.e. satisfy the abstract unique continuation property

$$
\operatorname{ker}\left(A^{*}+C_{t}-s\right) \cap D_{\min }=\{0\} .
$$

Clearly, the domains $D_{\max }$ and $D_{\min }$ are unchanged by the perturbation $C_{t}$ for any $t$. So, $\boldsymbol{\beta}$ does not depend on the parameter $t$. Moreover, the symplectic form $\omega$ is invariantly defined on $\boldsymbol{\beta}$ and so also independent of $t$. It follows (see [2], Theorem 3.9) that $\left\{\gamma\left(S_{t}\right)\right\}$ is continuous in $\mathcal{F} \mathcal{L}_{\gamma(D)}$.

With that, the family $\left\{A_{D}+C_{t}\right\}$ can be considered at the same time in the symmetric category, defining a spectral flow, and in the symplectic category, defining a Maslov index. Under the preceding assumptions the main result obtainable at that abstract level is the following abstract spectral flow formula (proved in [2], Theorem 5.1):

Theorem 6.4. Let $A_{D}$ be a self-adjoint extension of $A$ with compact resolvent (according to Assumption 6.2) and let $\left\{A_{D}+C_{t}\right\}$ be a family satisfying Assumption 6.3. Then

$$
\text { sf }\left\{A_{D}+C_{t}\right\}=\operatorname{mas}\left(\gamma\left(S_{t}\right), \gamma(D)\right) .
$$

The famous Agranovič-Dynin Theorem of index theory expresses the difference between two indices of the same elliptic operator over a manifold with boundary but with different boundary value conditions by the index of an induced operator living on the boundary (see e.g. Booss-Bavnbek and Wojciechowski [4]). For families we obtain a similar formula which involves the Hörmander index.

Let $D_{\min } \subset D, D^{\prime} \subset D_{\max }$ be two domains such that both $\left\{A_{D}+\right.$ $\left.C_{t}\right\}$ and $\left\{A_{D^{\prime}}+C_{t}\right\}$ become families of closed self-adjoint Fredholm operators. Then, in particular, the two spectral flows sf $\left(\left\{A_{D}+C_{t}\right\}\right)$ and $\mathbf{s f}\left(\left\{A_{D^{\prime}}+C_{t}\right\}\right)$ are well-defined integers. We are interested in 
the error when replacing one domain $D$ by another one $D^{\prime}$. From Definition 5.2, the following theorem follows. It expresses this error by a corresponding Hörmander index.

\section{Theorem 6.5. If}

$$
\operatorname{dim} \gamma(D) / \gamma(D) \cap \gamma\left(D^{\prime}\right)=\operatorname{dim} \gamma\left(D^{\prime}\right) / \gamma(D) \cap \gamma\left(D^{\prime}\right)<+\infty,
$$

then

$$
\mathbf{s f}\left(\left\{A_{D}+C_{t}\right\}\right)-\mathbf{s f}\left(\left\{A_{D^{\prime}}+C_{t}\right\}\right)=\sigma_{\text {Hör }}\left(\gamma\left(S_{0}\right), \gamma\left(S_{1}\right) ; \gamma(D), \gamma\left(D^{\prime}\right)\right) \text {. }
$$

Remark 6.6. (a) If there exists a domain $D^{\prime}$ such that $\mathbf{s f}\left(\left\{A_{D^{\prime}}+\right.\right.$ $\left.\left.C_{t}\right\}\right)$ vanishes, the preceding formula gives without any calculation the qualitative information that $\mathbf{s f}\left(\left\{A_{D}+C_{t}\right\}\right)$ only depends on the Cauchy data at the endpoints of the curve.

(b) If the family $\left\{C_{t}\right\}$ is a loop, then the Hörmander index vanishes and we obtain under the assumptions of Theorem 6.5 , not very surprisingly, that the spectral flow does not depend of the choice of the domain. Actually, it must vanish by topological considerations.

\section{General Boundary Data and Cauchy Data Spaces IN Distribution SPACE}

We shall apply the abstract spectral flow formula of Theorem 6.4 to the concrete situation of elliptic differential operators of first order over Riemannian manifolds. We begin by describing the embedding of the space $\boldsymbol{\beta}$ of abstract boundary data in a distribution space.

Throughout this section, $M$ denotes a fixed smooth compact connected Riemannian manifold of dimension $m$ with boundary $\partial M=\Sigma$. We shall work in the real category. So, let $E \rightarrow M$ be a smooth real vector bundle over $M$ of fibre dimension $n$. We fix the volume element on the manifold and the inner product in the bundle. We consider a symmetric linear elliptic differential operator of first order,

$$
A: C^{\infty}(M ; E) \longrightarrow C^{\infty}(M ; E)
$$

To be precise, the space $C^{\infty}(M ; E)$ consists of sections of the bundle $E$ which are smooth in the interior $M^{0}=M \backslash \Sigma$ and can be extended smoothly in a neighbourhood of $M$, when $M$ is embedded in an open manifold $M^{\prime} \supset M$ and $E$ the restriction of a smooth vector bundle $E^{\prime} \rightarrow M^{\prime}$. Let

$$
C_{0}^{\infty}(M ; E):=\left\{u \in C^{\infty}(M ; E) \mid \operatorname{supp} u \subset M^{0}\right\}
$$


and let $H^{s}(M ; E)$ denote the Sobolev space of order $s \in \mathbf{R}$.

Recall that $A$ symmetric means that the operator

$$
A_{0}:=\left.A\right|_{C_{0}^{\infty}(M ; E)}: C_{0}^{\infty}(M ; E) \rightarrow L_{2}(M ; E)
$$

is densely defined and symmetric in $L_{2}(M ; E)$.

Example 7.1. Let $E \rightarrow M$ be a bundle of Clifford modules with Clifford multiplication $c$ and compatible connection $\nabla$. Then the corresponding Dirac operator (considered as a real operator) satisfies our assumption.

Definition 7.2. We define

$$
\begin{array}{ll}
A_{\min }:=\overline{A_{0}} & \text { the minimal closed extension of } A_{0}, \\
A_{\max }:=\left(A_{0}\right)^{*} & \text { the adjoint of } A_{0} .
\end{array}
$$

Clearly, $A_{\max }$ is the maximal closed extension. We have

$$
A_{0} \subset A_{\min } \subset A_{\max }
$$

with

$$
D_{\min }:=\operatorname{dom}\left(A_{\min }\right)={\overline{C_{0}^{\infty}(M ; E)}}^{\mathcal{G}}={\overline{C_{0}^{\infty}(M ; E)}}^{H^{1}(M ; E)}
$$

and

$$
\begin{aligned}
D_{\max }:=\operatorname{dom}\left(A_{\max }\right)=\left\{u \in L_{2}(M ; E) \mid A u \in L_{2}(M ; E)\right. \\
\text { in the sense of distributions }\} .
\end{aligned}
$$

Here the superscript $\mathcal{G}$ means the closure in the graph norm which coincides with the 1 st Sobolev norm on $C_{0}^{\infty}(M ; E)$. In the same way, the superscript $H^{1}(M ; E)$ means the closure in the first Sobolev norm.

Remark 7.3. We shall emphasize that on a closed manifold $X$ any symmetric differential operator $A$ is essentially self-adjoint. So, there is only one self-adjoint $L_{2}(X ; E)$ extension $A_{D}$ of $A$. For an operator of first order, this extension is given by the domain $D:=D_{\min }=D_{\max }=$ $H^{1}(X ; E)$. In this case, the graph norm and the 1st Sobolev norm coincide on $H^{1}(X ; E)$ by the a priori estimate

$$
\|u\|_{1} \leq C\left(\|A u\|_{0}+\|u\|_{0}\right) \quad \text { for all } u \in D .
$$

We return to our compact manifold with boundary. We describe the space $\boldsymbol{\beta}$ of abstract boundary data (introduced in Section 6) as a subspace of the distribution space $H^{-\frac{1}{2}}\left(\Sigma ;\left.E\right|_{\Sigma}\right)$. We recall 
Proposition 7.4. Let $u \in L_{2}(M ; E)$ and $A u \in L_{2}(M ; E)$ (where $A u$ is defined in the distributional sense). Then the trace $\gamma(u)$ on $\Sigma$ is well defined as an element in $H^{-\frac{1}{2}}\left(\Sigma ;\left.E\right|_{\Sigma}\right)$. Moreover, there exists a constant $C$ independent of $u$ such that

$$
\|\gamma(u)\|_{-\frac{1}{2}} \leq C\left(\|A u\|_{0}+\|u\|_{0}\right)
$$

In particular we have a bounded operator

$$
\gamma: D_{\max } \longrightarrow H^{-\frac{1}{2}}\left(\Sigma ;\left.E\right|_{\Sigma}\right) .
$$

Proofs can be found e.g. in Booss-Bavnbek and Wojciechowski [4], Theorems 13.1 and 13.8 for our situation ( $A$ is of order 1) and in Hörmander [10] in greater generality (Theorem 2.2.1 and the Estimate (2.2.8), p. 194).

Remarks 7.5. (a) According to Proposition 7.4, there is a natural bounded embedding

$$
i: \beta \longrightarrow H^{-\frac{1}{2}}\left(\Sigma ;\left.E\right|_{\Sigma}\right)
$$

with $i(\boldsymbol{\beta})$ dense in $H^{-\frac{1}{2}}\left(\Sigma ;\left.E\right|_{\Sigma}\right)$ since $i(\boldsymbol{\beta}) \supset C^{\infty}\left(\Sigma ;\left.E\right|_{\Sigma}\right)$. Note that the whole space $H^{\frac{1}{2}}\left(\Sigma ;\left.E\right|_{\Sigma}\right)$ belongs to $i(\boldsymbol{\beta})$ because $H^{1}(M ; E) \subset D_{\max }$ and the restriction $\gamma: H^{1}(M ; E) \rightarrow H^{\frac{1}{2}}\left(\Sigma ;\left.E\right|_{\Sigma}\right)$ is surjective. Hence

$$
H^{\frac{1}{2}}\left(\Sigma ;\left.E\right|_{\Sigma}\right) \hookrightarrow i(\boldsymbol{\beta}) \hookrightarrow H^{-\frac{1}{2}}\left(\Sigma ;\left.E\right|_{\Sigma}\right)
$$

Also the left inclusion is dense. To see that, we recall from Ralston [16], Remark 2.1 and Remark 2.2 that there always exists a self-adjoint extension $A_{D}$ with domain $D \subset H^{1}(M ; E)$. Now, $\gamma(D)$ is a Lagrangian subspace of $\boldsymbol{\beta}$ (see the reference given in Section 6 after Assumption 6.2) and $\gamma(D) \subset \gamma\left(H^{1}(M ; E)\right) \subset \boldsymbol{\beta}$. So, the space $\gamma\left(H^{1}(M ; E)\right)$ is coisotropic in $\beta$. By Green's formula we can show that the annihilator $\gamma\left(H^{1}(M ; E)\right)^{0}=\{0\}$, so $\gamma\left(H^{1}(M ; E)\right)$ is dense in $\boldsymbol{\beta}$. This implies that $H^{\frac{1}{2}}\left(\Sigma ;\left.E\right|_{\Sigma}\right)=i \circ \gamma\left(H^{1}(M ; E)\right)$ is dense in $i(\beta)$.

(b) The embedding $i$ is not surjective, and the topology of $\beta$ is stronger than the topology of $H^{-\frac{1}{2}}\left(\Sigma ;\left.E\right|_{\Sigma}\right)$.

(c) On the function subspace $i(\boldsymbol{\beta}) \cap L_{2}\left(\Sigma ;\left.E\right|_{\Sigma}\right)$ of the distribution space $i(\boldsymbol{\beta})$, we can describe $\boldsymbol{\beta}$ 's symplectic form $\boldsymbol{\omega}$ explicitly by

$$
\begin{aligned}
\omega(\gamma(u), \gamma(v))=\left(A^{*} u, v\right)-\left(u, A^{*} v\right) & \\
& =\int_{\Sigma}\left(\sigma_{1}(A)(x, d \tau) \circ i \circ \gamma(u)(x), i \circ \gamma(v)(x)\right) d_{\Sigma} x
\end{aligned}
$$

for $i \circ \gamma(u), i \circ \gamma(v) \in i(\boldsymbol{\beta}) \cap L_{2}\left(\Sigma ;\left.E\right|_{\Sigma}\right)$, where $\sigma_{1}(A)$ denotes the principal symbol of $A$ and $\tau$ the inward normal variable. So, $\sigma_{1}(A)(x, d \tau)$ with $x \in \Sigma$ defines the almost complex structure on $\boldsymbol{\beta}$. 
(d) Clearly, $D_{\max }$ is something between $H^{1}(M ; E)$ and $L_{2}(M ; E)$. For $\operatorname{dim} M>1$, the Hilbert space $H^{1}(M ; E)$ is a proper subspace of $D_{\max }$. (See also our cylinder calculations below in Example 7.16). Also, see this Remark (a), it is dense in $D_{\max }$ in the graph norm. Consequently, the 1st Sobolev norm and the graph norm do not coincide on $H^{1}(M ; E)$. In that respect, the case of a manifold with boundary differs radically from the case of a closed manifold discussed above (where the two norms are equivalent).

Example 7.6. There are interesting subspaces of $H^{1}(M ; E)$ where the graph norm and the 1st Sobolev norm do coincide. Consider, e.g. the self-adjoint Fredholm extensions which are provided by the following setting. Let $R$ be a pseudo-differential operator over $\Sigma$ of order 0 satisfying suitable conditions (see, e.g., [4], Chapter 18). Then we have an estimate of the form

$$
\|u\|_{1} \leq C\left(\|A u\|_{0}+\|u\|_{0}+\|R \circ i \circ \gamma(u)\|_{\frac{1}{2}}\right) \text { for } u \in H^{1}(M ; E),
$$

and defining a domain by

$$
D:=\left\{u \in H^{1}(M ; E) \mid R \circ i \circ \gamma(u)=0\right\},
$$

we obtain a self-adjoint operator $A_{D}=\left.A^{*}\right|_{D}$. On the domain we have an a priori estimate

$$
\|u\|_{1} \leq C\left(\|A u\|_{0}+\|u\|_{0}\right) \text { for } u \in D
$$

and so the equivalence of the graph norm and the first Sobolev norm follows on the space $D$.

More generally, we have

Proposition 7.7. Let $D \subset H^{1}(M ; E)$ and let $A_{D}$ denote the corresponding $L_{2}$-extension $\left.A^{*}\right|_{D}$. If

i: $D$ is closed in $H^{1}(M ; E)$, and

ii: $A_{D}$ is self-adjoint in $L_{2}(M ; E)$

then the graph norm and the 1 st Sobolev norm coincide on $D$ and $A_{D}$ : $D \rightarrow L_{2}(M ; E)$ is a Fredholm operator with compact resolvent.

The proposition is proved by making use of the Open Mapping Theorem.

Note. Assertion (i) does not follow from assertion (ii). Neither does it follow from (i) alone that $A_{D}$ is a closed operator in $\mathcal{H}:=L_{2}(M ; E)$. The reason is that $A_{D}$ is closed, if and only if $D$ is closed in $D_{\max }$ in the graph norm. 
We have to be careful: On one side, not any arbitrary closed subspace of $H^{1}(M ; E)$ is closed in $D_{\max }$ in the graph norm. On the other side, there exist many closed subspaces of $H^{1}(M ; E)$ which are also closed in $D_{\max }$ in the graph norm, but dense in $L_{2}(M ; E)$. Take, e.g., the domain $D$ of (7.5) in Example 7.6 which is defined by a certain type of pseudo-differential projections over the boundary. Then it is well known that $A_{D}$ is a closed extension of $A$ (see e.g. Booss-Bavnbek and Wojciechowski [4], Lemma 20.1).

The following lemma is well known (though in different and in the view of the present authors not always completely correct formulation) and can be proved in different ways (e.g. [4], see also [2]).

Lemma 7.8. Let $\gamma: D_{\max } \rightarrow \boldsymbol{\beta}$ denote the canonical projection of $D_{\max }$ onto the factor space $\beta$. Let $\gamma(S)$ denote the Cauchy data space defined by $S:=\operatorname{ker} A_{\max }$. Let $D \subset D_{\max }$ be chosen as in Proposition 7.7. Then the pair $(\gamma(S), \gamma(D))$ is a Fredholm pair of Lagrangian subspaces of the symplectic Hilbert space $\boldsymbol{\beta}$.

Clearly, $D_{\max }$ and $D_{\min }$ are $C^{\infty}(M)$-modules, and hence we have

Lemma 7.9. The space $\beta$ is a $C^{\infty}(\Sigma)$-module.

Remark 7.10. The preceding lemma shows that $\beta$ is in the following sense local: If $\Sigma$ decomposes into $r$ connected components $\Sigma=\Sigma_{1} \sqcup$ $\cdots \sqcup \Sigma_{r}$, then $\boldsymbol{\beta}$ decomposes into

$$
\boldsymbol{\beta}=\bigoplus_{j=1}^{r} \boldsymbol{\beta}_{j}
$$

where

$$
\boldsymbol{\beta}_{j}:=\gamma\left(\left\{u \in D_{\max } \mid \operatorname{supp} u \subset \mathcal{N}_{j}\right\}\right),
$$

with a suitable collar neighbourhood $\mathcal{N}_{j}$ of $\Sigma_{j}$. Note that $\boldsymbol{\beta}_{j}$ is also a symplectic subspace of $\boldsymbol{\beta}$.

Let $\partial M=\Sigma$ consist of more than one connected component and let $\Sigma_{0} \cup \Sigma_{1}=\Sigma$ be a disjoint closed partition of $\Sigma$. Let $\beta=\beta_{0} \times$ $\boldsymbol{\beta}_{1}$ denote the corresponding decomposition of the symplectic Hilbert space $\boldsymbol{\beta}$. Assuming a slightly sharpened unique continuation property, namely that we have unique continuation of any solution from any single connected component of the boundary (and not only from the boundary as a whole as required else in this article), we obtain an interesting description of the Cauchy data space $\gamma(S)$ in the product 
space $\boldsymbol{\beta}_{0} \times \boldsymbol{\beta}_{1}$ : it is transversal to the factors $\boldsymbol{\beta}_{0} \times\{0\}$ and $\{0\} \times$ $\boldsymbol{\beta}_{1}$; its images $\pi_{j}(\gamma(S)) \subset \boldsymbol{\beta}_{j}$ under projection are dense; and it can be represented as the graph of a densely defined closed operator $T$ : $\pi_{0}(\gamma(S)) \rightarrow \boldsymbol{\beta}_{1}$.

More precisely and more generally, we consider two symplectic Hilbert spaces $\left\{H_{i}, \omega_{i}, J_{i},(\cdot, \cdot)_{i}\right\}_{i=0,1}$ and construct the product space $\mathcal{H}=$ $H_{0} \times H_{1}$ with the symplectic form

$$
\Omega\left(\langle x, y\rangle,\left\langle x^{\prime}, y^{\prime}\right\rangle\right)=\omega_{0}\left(x, x^{\prime}\right)+\omega_{1}\left(y, y^{\prime}\right) .
$$

Let $\lambda \subset \mathcal{H}$ be a Lagrangian subspace and

$$
\pi_{0}(\lambda):=\left\{x \in H_{0} \mid \exists y \in H_{1} \text { such that }\langle x, y\rangle \in \lambda\right\} .
$$

We make two assumptions,

1. $\lambda \cap H_{0} \times\{0\}=\{0\}$;

2. $\lambda \cap\{0\} \times H_{1}=\{0\}$.

From (2) we have an operator $T_{\lambda}: \pi_{0}(\lambda) \rightarrow H_{1}$ such that $\lambda$ becomes the graph of $T_{\lambda}$.

Lemma 7.11. The space $\pi_{0}(\lambda)$ is dense in $H_{0}$ and so $T_{\lambda}$ is a densely defined closed operator.

Proof. Let $a \perp \pi_{0}(\lambda)$. Then

$$
\Omega(\langle J a, 0\rangle,\langle x, y\rangle)=\omega_{0}(J a, x)=-(a, x)_{0}=0 \quad \text { for all }\langle x, y\rangle \in \lambda .
$$

Hence $\langle J a, 0\rangle \in \lambda$, and by assumption (1) this implies $a=0$. It follows that $\pi_{0}(\lambda)$ is at least dense.

We can give a more precise description of the embedding of $\boldsymbol{\beta}$ in the distribution space $H^{-\frac{1}{2}}\left(\Sigma:\left.E\right|_{\Sigma}\right)$ under the following assumption.

Assumption 7.12. We assume that the operator $A$ can be written in the form

$$
\left.A\right|_{\mathcal{N}}=\sigma\left(\frac{\partial}{\partial \tau}+B\right)
$$

in a collar neighbourhood $\mathcal{N}$ in $M$ of the boundary $\Sigma$. Here $\tau$ denotes the inward normal variable, the collar $\mathcal{N}$ is identified with $[0, \varepsilon) \times \Sigma$, the bundle $\left.E\right|_{\tau \times \Sigma}$ is identified with $\left.E\right|_{\Sigma}$. Moreover, $\sigma$ is a unitary bundle endomorphism of $\left.E\right|_{\Sigma}$ and $B$ a self-adjoint elliptic differential operator of first order acting on sections of $\left.E\right|_{\Sigma}$ over $\Sigma$.

This assumption shall be made in the rest of this section. It is satisfied for operators of Dirac type if all structures are product near the boundary. 
Let $\left\{\varphi_{k}, \lambda_{k}\right\}$ be a spectral resolution of $L_{2}(\Sigma)$ by eigensections of $B$. (Here and in the following we suppress mentioning the bundle $E$ ).

Assumption 7.13. For simplicity we assume $\operatorname{ker} B=\{0\}$.

Then

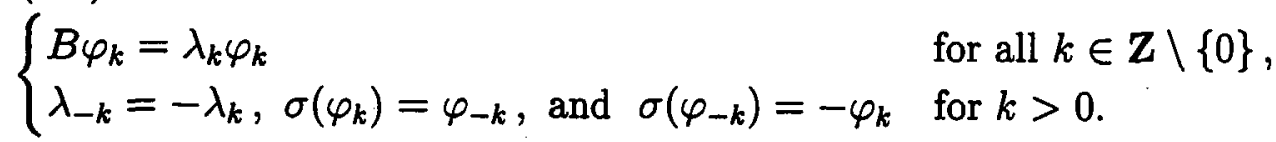

Example 7.14. A prominent self-adjoint Fredholm extension of $A$ is given by posing the so-called Atiyah-Patodi-Singer boundary condition

$$
D_{\text {aps }}:=\left\{u \in H^{1}(M) \mid \Pi_{>}\left(\left.u\right|_{\Sigma}\right)=0\right\} .
$$

Here $\Pi_{>}$denotes the pseudo-differential operator of order 0 which acts like the orthogonal projection of $L_{2}(\Sigma)$ onto the subspace spanned by the eigensections corresponding to the positive eigenvalues. Clearly

$$
\gamma\left(D_{\text {aps }}\right)={\overline{\left[\left\{\varphi_{k}\right\}_{k<0}\right]}}^{H^{\frac{1}{2}}(\Sigma)} .
$$

(For easier presentation we do not write the symbol $i$ for the embedding of $\boldsymbol{\beta}$ into $\left.H^{-\frac{1}{2}}(\Sigma)\right)$.

We shall describe the space of abstract boundary data as a 'graded' subspace of the distribution space $H^{-\frac{1}{2}}(\Sigma)$.

Proposition 7.15. We have

$$
\boldsymbol{\beta}={\overline{\left[\left\{\varphi_{k}\right\}_{k<0}\right]}}^{H^{\frac{1}{2}}(\Sigma)} \oplus{\overline{\left[\left\{\varphi_{k}\right\}_{k>0}\right]}}^{H^{-\frac{1}{2}}(\Sigma)} .
$$

Note that the decomposition of $\beta$ into a $H^{\frac{1}{2}}(\Sigma)$-part and a $H^{-\frac{1}{2}}(\Sigma)-$ part is not unique. Here we have chosen a partitioning of the eigenvalues at zero. But any other partitioning with one accumulation point at $-\infty$ and one at $+\infty$ would do. The difference between the various representations, however, is only finite-dimensional.

Proof. Let $u \in{\overline{\left[\left\{\varphi_{k}\right\}_{k>0}\right]}}^{H^{-\frac{1}{2}}(\Sigma)}$, i.e.

$$
u=\sum_{k>0} a_{k} \varphi_{k} \quad \text { with } \quad \sum_{k>0}\left|a_{k}\right|^{2} / \lambda_{k}<\infty .
$$

On the neck $\mathcal{N}$ we consider the section

$$
\widetilde{u}(\tau, y):=\sum_{k>0} a_{k} e^{-\lambda_{k} \tau} \otimes \varphi_{k}(y) .
$$


Then

$$
(\widetilde{u}, \widetilde{u}) \leq \sum_{k>0}\left|a_{k}\right|^{2} \int_{0}^{\varepsilon} e^{-2 \lambda_{k} \tau} d \tau=\sum_{k>0}\left|a_{k}\right|^{2} \frac{1-e^{-2 \lambda_{k} \varepsilon}}{2 \lambda_{k}} \leq \frac{1}{2} \sum_{k>0} \frac{\left|a_{k}\right|^{2}}{\lambda_{k}} .
$$

So, $\tilde{u} \in L_{2}(\mathcal{N})$ and $A \widetilde{u}=0$. We choose a smooth cut-off function $\chi$ with support in the neck $\mathcal{N}$. Then $\chi \widetilde{u} \in L_{2}(M)$ and $\gamma(\chi \widetilde{u})=u$. So we have that

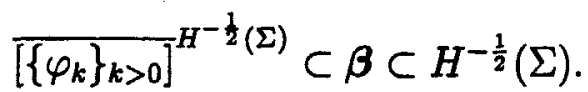

As noticed before in Remark 7.5.a, we know that the whole space $H^{\frac{1}{2}}(\Sigma)$ is contained in $\boldsymbol{\beta}$. It remains to show that the spaces

$$
\lambda:={\overline{\left[\left\{\varphi_{k}\right\}_{k<0}\right]}}^{H^{\frac{1}{2}}(\Sigma)} \quad \text { and } \quad \mu:={\overline{\left[\left\{\varphi_{k}\right\}_{k>0}\right]}}^{H^{-\frac{1}{2}}(\Sigma)}
$$

span the whole $\boldsymbol{\beta}$. By Lemma 7.8, $\lambda$ is a Lagrangian subspace of $\boldsymbol{\beta}$. By definition, $\mu$ is closed in $H^{-\frac{1}{2}}(\Sigma)$. But $\beta \hookrightarrow H^{-\frac{1}{2}}(\Sigma)$ is continuous, so $\mu$ is closed in $\boldsymbol{\beta}$. By (7.8) that implies $\sigma(\mu)=\gamma(\lambda)$. As noticed in Remark 7.5.c, our $\sigma$ yields the almost complex structure on $\boldsymbol{\beta}$. So, $\mu=\sigma(\lambda)$ and $\mu$ must be Lagrangian subspace of $\boldsymbol{\beta}$. Hence, $\boldsymbol{\beta}=\lambda \oplus \mu$.

Example 7.16. Based on the preceding embedding of $\beta$ in the distribution space $H^{-\frac{1}{2}}(\Sigma)$ we shall explain the embedding of the Cauchy data spaces. An instructive example is provided by the cylinder $M=$ $I \times \widehat{\Sigma}$ with $I=[0,1]$ and $\partial M=-\widehat{\Sigma} \sqcup \widehat{\Sigma}=: \Sigma$. Let $\widehat{E} \rightarrow \widehat{\Sigma}$ be a real vector bundle and define $E:=\pi^{*} \widehat{E}$ where $\pi: I \times \widehat{\Sigma} \rightarrow \widehat{\Sigma}$ is the natural projection. Let $A=\sigma\left(\frac{\partial}{\partial \tau}+B\right)$. Here $\tau$ denotes the variable running in $I, B$ is a self-adjoint elliptic differential operator acting on sections of $\widehat{E}$ over $\widehat{\Sigma}$, and $\sigma$ is a skew-symmetric bundle endomorphism which is an anti-involution and anti-commutes with $B$.

For simplicity, we assume that $B$ is invertible. Let $\left\{\varphi_{k}\right\}$ denote the system of eigensections with corresponding eigenvalues $\left\{\lambda_{k}\right\}$. We have the same relations as listed in (7.8) (be aware, though, that our present $B$ is only defined over one component of the boundary, namely $\widehat{\Sigma}$ and not over the whole boundary $-\widehat{\Sigma} \sqcup \widehat{\Sigma}$ ).

As explained in Remark 7.10.a, the space of boundary data splits $\boldsymbol{\beta}=\boldsymbol{\beta}_{0} \times \boldsymbol{\beta}_{1}$, and we have by Proposition 7.15 (notice the reversed orientation at the ends)

$$
\begin{aligned}
& \boldsymbol{\beta}_{0}={\overline{\left[\left\{\varphi_{k}\right\}_{k<0}\right]}}^{H^{\frac{1}{2}}(\widehat{\Sigma})} \oplus{\overline{\left[\left\{\varphi_{k}\right\}_{k>0}\right]}}^{H^{-\frac{1}{2}}(\hat{\Sigma})} \text { and }
\end{aligned}
$$

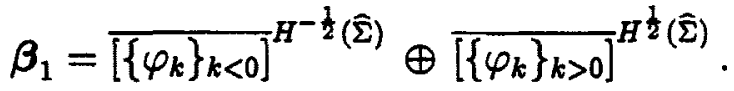


We shall characterize the Cauchy data space $\gamma\left(S^{\text {cyl }}\right)$ with $S^{\text {cyl }}:=$ $\operatorname{ker} A^{*}$. Let $u \in S^{\mathrm{cyl}}$, so $u \in L_{2}(M)$ and $A u=0$ in the distributional sense. Then $u$ must take the form

$$
u(\tau, y):=\sum_{k \neq 0} a_{k} e^{-\lambda_{k} \tau} \otimes \varphi_{k}(y),
$$

and $u \in L_{2}(M)$ implies

$$
\sum_{k \neq 0}\left|a_{k}\right|^{2} \int_{0}^{1} e^{-2 \lambda_{k} \tau} d \tau=\sum_{k \neq 0}\left|a_{k}\right|^{2} \frac{1-e^{-2 \lambda_{k}}}{2 \lambda_{k}}<+\infty .
$$

We consider the distribution $v:=\gamma(u)=\left(v_{0}, v_{1}\right) \in \boldsymbol{\beta}_{0} \oplus \boldsymbol{\beta}_{1} \subset H^{-\frac{1}{2}}(\widehat{\Sigma}) \times$ $H^{-\frac{1}{2}}(\widehat{\Sigma})$. We have

$$
v_{0}=\sum_{k<0} a_{k} \varphi_{k}+\sum_{k>0} a_{k} \varphi_{k}=v_{0}^{-}+v_{0}^{+}
$$

with coefficients satisfying conditions equivalent to (7.11). So

$$
\sum_{k<0}\left|a_{k}\right|^{2} \frac{e^{2\left|\lambda_{k}\right|}}{\left|\lambda_{k}\right|}<+\infty \text { and } \sum_{k>0}\left|a_{k}\right|^{2} / \lambda_{k}<+\infty .
$$

It follows from the estimates that $v_{0}^{-} \in C^{\infty}(\widehat{\Sigma})$ and $v_{0}^{+} \in H^{-\frac{1}{2}}(\widehat{\Sigma})$. One notices that the preceding estimate for the coefficients of $v_{0}$ is stronger than the assertion. that $\sum_{k<0}\left|a_{k}\right|^{2}\left|\lambda_{k}\right|^{N}<+\infty$ for all natural $N$. So, our estimates confirm that not each smooth section can appear as initial value over $\{0\} \times \widehat{\Sigma}$ of a solution of $A^{*} u=0$ over the cylinder.

Similarly, we see that $v_{1}$ is written as

$$
v_{1}=\sum_{k \neq 0} a_{k} e^{-\lambda_{k}} \varphi_{k}=\sum_{k<0} a_{k} e^{-\lambda_{k}} \varphi_{k}+\sum_{k>0} a_{k} e^{-\lambda_{k}} \varphi_{k}=v_{1}^{-}+v_{1}^{+},
$$

with $v_{1}^{-} \in H^{-\frac{1}{2}}(\widehat{\Sigma})$ and $v_{1}^{+} \in C^{\infty}(\widehat{\Sigma})$.

It follows that the Cauchy data space $\gamma\left(S^{\text {cyl }}\right)$ can be written as the graph of an unbounded, densely defined, closed operator $T: \operatorname{dom} T \rightarrow$ $\boldsymbol{\beta}_{1}$, mapping $v_{0} \mapsto v_{1}$ with $\operatorname{dom} T \subset \boldsymbol{\beta}_{0}$. (See also Lemma 7.11).

Without proof we mention that, moreover,

$$
\gamma\left(S^{\mathrm{cyl}}\right) \cap \gamma\left(D_{\text {aps }}^{\mathrm{cyl}}\right)=\{0\},
$$

where $D_{\mathrm{aps}}^{\text {cyl }}$ denotes the corresponding Atiyah-Patodi-Singer domain.

Note that the $H^{\frac{1}{2}}(\Sigma)$-part of $\gamma(S)$ is not closed in $L_{2}(\Sigma)$ because of the condition (7.12), left side, on the coefficients. Hence, $\gamma(S) \cap L_{2}(\Sigma)$ is not closed in $L_{2}(\Sigma)$ (recall $\Sigma=-\widehat{\Sigma} \sqcup \widehat{\Sigma}$ ). 
Remark 7.17. (a) We can easily drop the Assumption 7.13: For singular $B$, we notice that ker $B$ is finite-dimensional and has a symplectic structure. So any decomposition of $\operatorname{ker} B$ into two complementary Lagrangian subspaces $\mu_{-}, \mu_{+}$permits to carry through all the preceding arguments about the splitting of $\beta$ when we add $\mu_{+}$to the linear closure $\left[\left\{\varphi_{k}\right\}_{k>0}\right]$, and $\mu_{-}$to $\left[\left\{\varphi_{k}\right\}_{k<0}\right]$.

(b) For a related investigation of $\boldsymbol{\beta}$ in the cylinder case see also Brüning, Lesch [5].

\section{Generalized Yoshida-Nicolaescu Formula in $H^{-\frac{1}{2}}(\Sigma)$}

In the preceding section, we have seen how to embed the space $\beta$ of abstract boundary data into the distribution space $H^{-\frac{1}{2}}(\Sigma)$ in the concrete situation of a symmetric elliptic differential operator $A$ over a compact connected smooth Riemannian manifold $M$ with boundary $\Sigma$.

Now we must discuss the Assumptions 6.2 and 6.3 for establishing concrete versions of our abstract spectral flow formula.

Assumption 6.2 demands that $A$ admits a self-adjoint 'elliptic' boundary condition in the sense that a domain $D \subset D_{\max }$ exists with $\gamma(D)$ Lagrangian subspace of the symplectic Hilbert space $\boldsymbol{\beta}$ of abstract boundary data and that $A_{D}:=\left.A^{*}\right|_{D}$ has a compact resolvent. Hence the operators $A_{D}+C_{t}$ are self-adjoint (unbounded) Fredholm operators, where $\left\{C_{t}\right\}_{t \in I}$ is a continuous family of symmetric bundle homomorphisms.

Additionally, we shall assume $D \subset H^{1}(M)$,

Definition 8.1. In the following, domains which satisfy Assumption 6.2 and belong to $H^{1}(M)$ will be called general self-adjoint elliptic boundary conditions.

Many examples of such domains are given in Booss-Bavnbek and Wojciechowski [4] with emphasis on domains defined by pseudo-differential projections $P$ over the boundary which have the same principal symbol as the Atiyah-Patodi-Singer projection and satisfy the symmetry relation $\sigma(\operatorname{Id}-P) \sigma^{*}=P$ (see the Examples 7.6 and 7.14). Below we will meet, however, also general elliptic boundary conditions which are of different type, namely relating data from different connected components of the boundary pointwise and hence they are truly global.

Assumption 6.3 requires that all operators $A+C_{t}-s$ (for small $|s|$ ) satisfy the unique continuation property $\operatorname{ker}\left(A^{*}+C_{t}-s\right) \cap D_{\min }=\{0\}$. 
As discussed in the preceding section, we have

$$
D_{\min }={\overline{C_{0}^{\infty}}(M \backslash \Sigma)}^{H^{1}(M)}=H_{0}^{1}(M \backslash \Sigma) \text {. }
$$

So, Assumption 6.3 requires that any solution which vanishes on the whole boundary vanishes on the whole manifold. For first order elliptic operators on connected manifolds this follows from the weak unique continuation property (if satisfied), namely that any solution, which vanishes on an open subset, must vanish on the whole connected component of the manifold. As shown in Plis [15], that property is not always satisfied. However, if $A$ is an operator of Dirac type, also $A+C_{t}-s$ is such an operator. For all such operators the weak unique continuation property is proven in [4]. More generally, it is shown there that any elliptic operator of first order with self-adjoint 'tangential' operator up to a perturbation of order 0 , satisfies the weak unique continuation property.

Assumption 8.2. In the following we shall restrict ourselves to elliptic differential operators of the form $A+C_{t}$ which satisfy the weak unique continuation property.

Let $\gamma\left(S_{t}\right)$ denote the abstract Cauchy data space of the operator $A+C_{t}$, where $S_{t}:=\operatorname{ker}\left(A^{*}+C_{t}\right)$. Theorem 6.4 (for details see [2]) yields at once

Theorem 8.3. Under the preceding assumptions the spectral flow of the family $\left\{A_{D}+C_{t}\right\}$ is well defined. Moreover, the family $\left\{\gamma\left(S_{t}\right)\right\}$ is a continuous curve in the Fredholm Lagrangian Grassmannian $\mathcal{F} \mathcal{L}_{\gamma(D)}$ and we have

$$
\text { sf }\left\{A_{D}+C_{t}\right\}=\operatorname{mas}\left(\left\{\gamma\left(S_{t}\right)\right\}, \gamma(D)\right)
$$

We have two corollaries for the spectral flow on closed manifolds with fixed hypersurface. The first corollary treats the case of a separating hypersurface, the second the case of a non-separating hypersurface. Both cases shall be reduced to the situation of the preceding theorem by cutting the manifold along $\Sigma$. Then we receive a manifold with two isometric boundary components in both cases.

First, let $M$ be a connected partitioned manifold $M=M_{-} \cup_{\Sigma} M_{+}$ with $\Sigma=M_{-} \cap M_{+}=\partial M_{-}=\partial M_{+}$. Let $A$ be a symmetric elliptic differential operator of first order over $M$ acting on sections of a bundle $E$ and let $C_{t}$ be a continuous family of symmetric bundle endomorphisms. Let $A_{ \pm}$denote the restrictions of the operator $A$ to the part manifolds $M_{ \pm}$with the minimal domains $D_{\min }^{ \pm}$, the maximal domains $D_{\max }^{ \pm}$, and corresponding abstract boundary data spaces $\beta^{ \pm}$ 
and projections $\gamma_{ \pm}: D_{\max } \rightarrow \beta^{ \pm}$. We assume that the (weak) unique continuation property $\operatorname{ker}\left(A_{ \pm}^{*}+C_{t}\right) \cap D_{\min }^{ \pm}=\{0\}$ is satisfied on each part manifold.

Let $M_{\sharp}$ denote the compact manifold $M_{-} \sqcup M_{+}=M \backslash \Sigma \cup(-\Sigma \sqcup \Sigma)$ with boundary $\partial M_{\sharp}=\partial M_{-} \sqcup \partial M_{+}=-\Sigma \sqcup \Sigma$ and let $A_{\sharp}$ denote the induced operator over $M_{\sharp}$ with minimal and maximal domains $D_{\min }^{\sharp}, D_{\max }^{\sharp}$ and abstract boundary data space $\boldsymbol{\beta}^{\sharp}$. Fixing the separating hypersurface $\Sigma$ induces a decomposition $L_{2}(M) \cong L_{2}\left(M_{-}\right) \oplus$ $L_{2}\left(M_{+}\right)$. Correspondingly we obtain

$$
D_{\min }^{\sharp} \cong D_{\min }^{-} \oplus D_{\min }^{+} \text {and } D_{\max }^{\sharp} \cong D_{\max }^{-} \oplus D_{\max }^{+}
$$

for the minimal and maximal domains of $A_{\sharp}$ and

$$
\boldsymbol{\beta}^{\sharp}=D_{\text {max }}^{\sharp} / D_{\text {min }}^{\sharp} \cong D_{\text {max }}^{-} / D_{\text {min }}^{-} \oplus D_{\text {max }}^{+} / D_{\text {min }}^{+} \cong \boldsymbol{\beta}^{-} \oplus \boldsymbol{\beta}^{+} .
$$

Similarly we have a decomposition of the Cauchy data space

$$
\gamma\left(\operatorname{ker}\left(A_{\sharp}^{*}+C_{t}\right)\right) \cong \gamma_{-}\left(\operatorname{ker}\left(A_{-}^{*}+C_{t}\right)\right) \oplus \gamma_{+}\left(\operatorname{ker}\left(A_{+}^{*}+C_{t}\right)\right) .
$$

Over the manifold $M_{\sharp}$ there is a natural self-adjoint general elliptic boundary condition (in the sense of Definition 8.1) for $A_{\sharp}$ defined by the pasting domain

$$
D^{\sharp}:=\left\{u \in D_{\max }^{\sharp} \mid i_{-} \circ \gamma_{-}(u)=i_{+} \circ \gamma_{+}(u)\right\},
$$

where $i_{ \pm}: \boldsymbol{\beta}^{ \pm} \hookrightarrow H^{-\frac{1}{2}}(\Sigma)$ denote the inclusions.

Lemma 8.4. The pasting domain $D^{\sharp} \subset D_{\max }^{\sharp}$ over the cut manifold $M_{\sharp}$ can be naturally identified with the 1st Sobolev space $H^{1}(M)$ over the underlying closed manifold.

Proof. We sketch only the proof. So, let $u, A_{\sharp} u \in L_{2}\left(M_{\sharp}\right)$. By localizing in a bicollar neck $\mathcal{N}=[-1,1] \times \Sigma$ of $\Sigma$ in $M$ and explicit calculation of the Green formula we find for each $v \in C_{0}^{\infty}(\mathcal{N})$

$$
\begin{aligned}
(A u, v)= & \lim _{\varepsilon \rightarrow 0}\left\{\int_{\mathcal{N}} \int_{-1}^{-\varepsilon}((A u)(\tau, x), v(\tau, x)) d \tau d_{\Sigma} x\right. \\
& \left.\quad+\int_{\mathcal{N}} \int_{\varepsilon}^{1}((A u)(\tau, x), v(\tau, x)) d \tau d_{\Sigma} x\right\} \\
= & \cdots=(u, A v) .
\end{aligned}
$$

Since there are no other terms we conclude $\left.u\right|_{\mathcal{N}} \in H_{\text {loc }}^{1}(\mathcal{N})$, hence $u \in H^{1}(M)$. 
Since $A$ is elliptic of first order, we can identify $D^{\sharp}$ with the 1st Sobolev space $H^{1}(M)$. Because the Sobolev restriction map

$$
\gamma: H^{1}(M) \rightarrow H^{\frac{1}{2}}(\Sigma)
$$

is surjective we obtain

$$
\begin{aligned}
\gamma\left(D^{\sharp}\right) & =\left\{\left(\gamma_{-}(u), \gamma_{+}(u)\right) \mid u \in H^{1}(M)\right\} \\
& =\Delta\left(H^{\frac{1}{2}}(\Sigma) \times H^{\frac{1}{2}}(\Sigma)\right) \cong H^{\frac{1}{2}}(\Sigma),
\end{aligned}
$$

where $\Delta$ denotes the diagonal in the product space. Of course, the $H^{\frac{1}{2}-}$ diagonal $\Delta:=\Delta\left(H^{\frac{1}{2}}(\Sigma) \times H^{\frac{1}{2}}(\Sigma)\right)$ is not closed in $H^{-\frac{1}{2}}(\Sigma) \times H^{-\frac{1}{2}}(\Sigma)$ but it is closed in the symplectic Hilbert space $\boldsymbol{\beta}^{\sharp}$ which is a subspace of $H^{-\frac{1}{2}}(\Sigma) \times H^{-\frac{1}{2}}(\Sigma)$ (as discussed in the preceding section).

With all these notations we can rewrite the preceding theorem for the particular manifold $M_{\sharp}$ with boundary and obtain

Corollary 8.5. (Spectral Flow Formula of Yoshida-Nicolaescu Type for Partitioned Manifolds) Let $A$ be a symmetric elliptic differential operator of first order acting on sections of an Euclidean bundle over a closed connected Riemannian partitioned manifold $M=M_{-} \cup_{\Sigma} M_{+}$ with $\Sigma=M_{-} \cap M_{+}=\partial M_{-} \doteq \partial M_{+}$. Let $\left\{C_{t}\right\}$ be a continuous curve of symmetric bundle endomorphisms such that the weak unique continuation property is satisfied for each $A+C_{t}-s$ with small $|s|$. Then we have

$$
\text { sf }\left\{A+C_{t}\right\}=\operatorname{mas}\left(\left\{\gamma_{-}\left(S_{t}^{-}\right) \oplus \gamma_{+}\left(S_{t}^{+}\right)\right\}, \Delta\right),
$$

where $S_{t}^{ \pm}:=\operatorname{ker}\left(A_{ \pm}^{*}+C_{t}\right), \gamma_{ \pm}$denotes the projections from $D_{\max }^{ \pm}$ onto $\beta^{ \pm} \subset H^{-\frac{1}{2}}(\Sigma)$, and $\Delta$ denotes the diagonal in the product space $H^{\frac{1}{2}}(\Sigma) \times H^{\frac{1}{2}}(\Sigma)$.

A second corollary of Theorem 8.3 is obtained when the hypersurface $\Sigma$ does not separate $M$. We assume that the normal bundle of $\Sigma$ is oriented. We cut the manifold at $\Sigma$ and attach a copy of $\Sigma$ at each side. So, we obtain a new manifold

$$
M_{\sharp}=M \backslash \Sigma \cup(-\Sigma) \cup \Sigma
$$

with boundary $(-\Sigma) \cup \Sigma$. For the induced operator $A_{\sharp}+C_{t}$ over $M_{\sharp}$ we find that the minimal and maximal domains do not split in product form. However, the space of boundary data, being a $C^{\infty}\left(\partial M_{\sharp}\right)$-module splits into $\boldsymbol{\beta}^{\sharp}=\boldsymbol{\beta}^{-} \oplus \boldsymbol{\beta}^{+}$with the projections $\gamma_{ \pm}: D_{\max } \rightarrow \boldsymbol{\beta}^{ \pm}$and the inclusions $i_{ \pm}: \beta^{ \pm} \hookrightarrow H^{-\frac{1}{2}}(\Sigma)$. 
Over $M_{\sharp}$ we specify the domain

$$
D^{\sharp}:=\left\{u \in D_{\max } \mid i_{-} \circ \gamma_{-}(u)=i_{+} \circ \gamma_{+}(u)\right\},
$$

which can be identified with $H^{1}(M)$ (c.f. Lemma 8.4) and defines a general elliptic boundary condition in the sense of Definition 8.1.

Notice that the Cauchy data spaces $\gamma_{\sharp}\left(\operatorname{ker}\left(A_{\sharp}^{*}+C_{t}\right)\right)$ do not split (see Remark 7.10 and Example 7.16).

With all these notations we can rewrite the preceding theorem for the particular manifold $M_{\sharp}$ with boundary and obtain

Corollary 8.6. (Spectral Flow Formula of Yoshida-Nicolaescu Type for Non-Partitioned Manifolds) Let $A$ be a symmetric elliptic differential operator of first order acting on sections of a bundle over a closed connected Riemannian manifold $M$. Let $\Sigma$ be a hypersurface with orientable normal bundle. Let $\left\{C_{t}\right\}$ be a continuous curve of symmetric bundle endomorphisms of $E$ over $M$ such that the weak unique continuation property is satisfied for each $A+C_{t}-s$ for small $|s|$. Then we have

$$
\mathbf{s f}\left\{A+C_{t}\right\}=\operatorname{mas}\left(\left\{\gamma_{\sharp}\left(S_{t}^{\sharp}\right)\right\}, \Delta\right),
$$

where $S_{t}^{\sharp}:=\operatorname{ker}\left(A_{\sharp}^{*}+C_{t}\right), \gamma_{\sharp}=\left(\gamma_{-}, \gamma_{+}\right)$denotes the projection from $D_{\max }^{\sharp}$ onto $\beta^{\sharp}=\beta^{-} \oplus \beta^{+} \subset H^{-\frac{1}{2}}(\Sigma) \times H^{-\frac{1}{2}}(\Sigma)$, and $\Delta$ denotes the diagonal in the product space $H^{\frac{1}{2}}(\Sigma) \times H^{\frac{1}{2}}(\Sigma)$.

The Maslov indices in our formulas (8.1), (8.3), and (8.4) are defined in the distribution space $H^{-\frac{1}{2}}(\Sigma)$ because the boundary data and in particular the Cauchy data spaces naturally belong there. We shall now give a general existence theorem for a finite-dimensional symplectic reduction of the key formula (8.1) of Theorem 8.3. The reduction permits the calculation of the spectral flow via the Maslov index in function spaces over the hypersurface.

As in Theorem 8.3, we consider a symmetric elliptic differential operator $A$ of first order acting on sections of a bundle $E$ over a compact connected Riemannian manifold $M$ with boundary $\Sigma$ and a continuous curve $\left\{C_{t}\right\}$ of symmetric bundle endomorphisms of $E$ over $M$. Moreover, we fix a domain $D \subset H^{1}(M)$ which defines a general self-adjoint elliptic boundary condition (i.e., $A_{D}:=\left.A^{*}\right|_{D}$ has a compact resolvent, or, equivalently, the a priori estimate (7.6) is satisfied on $D$ ). Finally, we assume that the weak unique continuation property is satisfied for each $A+C_{t}-s$ for small $|s|$. 
Theorem 8.7. (a) Under the preceding assumptions, there exists a subspace $W \subset D$ such that $\gamma(W)$ is a closed subspace of $\gamma(D)$ of finite codimension which intersects all Cauchy data spaces $\gamma\left(S_{t}\right)$ transversally and with annihilator $\gamma(W)^{0}$ belonging to the function space $H^{\frac{1}{2}}(\Sigma)$ (under the embedding $\left.i: \boldsymbol{\beta} \rightarrow H^{-\frac{1}{2}}(\Sigma)\right)$.

(b) Moreover, we have

$$
\text { sf }\left\{A_{D}+C_{t}\right\}=\operatorname{mas}\left(\left\{\widetilde{\gamma\left(S_{t}\right)}\right\}, \widetilde{\gamma(D)}\right)_{\tilde{\boldsymbol{\beta}}}
$$

Here $S_{t}:=\operatorname{ker}\left(A^{*}+C_{t}\right), \gamma: D_{\max } \rightarrow \boldsymbol{\beta}$ denotes the projection, and $\widetilde{\boldsymbol{\beta}}$ denotes the finite-dimensional symplectic vector space $\gamma(W)^{0} / \gamma(W)$ with $\tilde{\lambda}:=\rho_{\gamma(W)}(\lambda)$ for $\lambda \in \boldsymbol{\beta}$ where

$$
\rho_{\gamma(W)}: \lambda \longrightarrow\left(\left(\lambda \cap \gamma(W)^{0}\right)+\gamma(W)\right) / \gamma(W)
$$

denotes the finite-dimensional reduction $\mathcal{F L}_{\gamma(W)}^{(0)} \rightarrow \operatorname{Lag}(\widetilde{\boldsymbol{\beta}})$, discussed in Proposition 4.5.

Proof. To begin with, we fix a parameter $t \in I$ and write for a moment shorthanded $A$ instead of $A+C_{t}$ and $S$ instead of $S_{t}$. Then

1. $A^{*}$ is surjective, i.e. $A^{*}\left(D_{\max }\right)=L_{2}(M)$ because of the unique continuation property and $A^{* *}=A_{\min }$.

2. The range $A(D)$ is closed in $L_{2}(M)$ and its orthogonal complement $A_{D}(D)^{\perp}$ in $L_{2}(M)$ is finite-dimensional because of the Fredholm property.

3. The restriction $\left.A^{*}\right|_{H^{1}(M)}$ of $A^{*}$ to the 1st Sobolev space is also surjective, i.e. $A^{*}\left(D_{\max }\right)=L_{2}(M)$ because of

$$
A^{*}(D) \subset A^{*}\left(H^{1}(M)\right) \stackrel{\text { dense }}{\longleftrightarrow} L_{2}(M)
$$

and the finite codimension of $A^{*}(D)$ in $L_{2}(M)$.

4. Consequently, there exists a finite-dimensional extension $\widetilde{W}$ of the domain $D$ in the 1st Sobolev space with $A_{\widetilde{W}}:=\left.A^{*}\right|_{\widetilde{W}}$ surjective; more precisely we have (i) $D \subset \widetilde{W} \subset H^{1}(M)$ with $\operatorname{dim} \widetilde{W} / D<$ $+\infty$, and (ii) $A^{*}(\widetilde{W})=L_{2}(M)$.

5. Finally, we see that $A_{\widetilde{W}}$ is a closed operator because, being only a finite-dimensional extension of $A_{D}$, it inherits the a priori estimate (7.6) from $D$.

We define

$$
W:=\left\{u \in D \mid\left(A_{\widetilde{W}} v, u\right)=\left(v, A_{D} u\right) \text { for all } v \in \widetilde{W}\right\},
$$

i.e. $A_{W}=\left(A_{\widetilde{W}}\right)^{*}$. Since $A_{\widetilde{W}}(\widetilde{W})=L_{2}(M)$, we have $S \cap W=\{0\}$. By taking the boundary values, we obtain $\gamma(W) \cap \gamma(S)=\{0\}$ by the 
assumed unique continuation property. Moreover, it follows $\gamma(W)^{0}=$ $\gamma(\widetilde{W})$, hence $\gamma(W)^{0} \subset H^{\frac{1}{2}}(\Sigma)$.

Until now, we have fixed the operator $A_{t}=A+C_{t}$. Now we consider the whole family. First we carry out the preceding construction for $t=0$ and obtain a space $W$ with the wanted properties at $t=0$. By the same argument as in the proof of Proposition 4.4 we obtain also an $\varepsilon>0$ such that $\gamma(W) \cap \gamma\left(S_{t}\right)=\{0\}$ for $0 \leq t<\varepsilon$. So, we repeat our construction and obtain a finite list $\widetilde{W}_{1}, \ldots, \widetilde{W}_{N} \subset H^{1}(M)$ of finitedimensional extensions of $D$ and corresponding spaces $W_{1}, \ldots, W_{N}$ as in (8.6). Then, in particular, we have for each $t \in I$ at least one $W_{i}$ such that $S_{t} \cap W_{i}=\{0\}$.

We define $W:=\cap_{i=1}^{N} W_{i} \subset D$. Then, still, $\gamma(W)^{0}=\sum \gamma\left(\widetilde{W}_{i}\right) \subset$ $H^{\frac{1}{2}}(\Sigma)$ and $S_{t} \cap W=\{0\}$ for all $t \in I$. That proves (a).

From (a) we obtain

$$
\widetilde{\boldsymbol{\beta}}:=\gamma(W)^{0} / \gamma(W) \cong \gamma(W)^{\perp} \cap \gamma(W)^{0} \subset H^{\frac{1}{2}}(\Sigma) .
$$

Now, the assertion (b) follows from (a) and Theorem 8.3 by Theorem 4.2.b and Remark 5.1.

Remarks 8.8. (a) According to Proposition 4.4, there exists always a domain $W \subset D$ such that $\gamma(W)$ is isotropic and of finite codimension and transversal with all $\gamma\left(S_{t}\right)$. The point in the preceding proof is, however, that we need $\gamma(W)^{0} \subset H^{\frac{1}{2}}(\Sigma)$.

(b) In (8.7) we have realized the factor space $\widetilde{\boldsymbol{\beta}}$ in a function space on

$\Sigma$. Correspondingly, the symplectic form $\widetilde{\omega}$ on $\widetilde{\beta}$ can also be written as

$$
\widetilde{\omega}(f, g)=\int_{\Sigma}\left(\sigma_{1}(A)(x, d \tau)(f(x)), g(x)\right) d_{\Sigma} x
$$

with true integration over a scalar product.

(c) If the operator is of product form near the boundary, there exists a more explicit and computable finite dimensional symplectic reduction, namely by taking adiabatic limits (see Yoshida [19] and Nicolaescu [12]). 


\section{Appendix A. A Characterization of Lagrangian FREDHOLM PAIRS}

Recall that the space of Fredholm pairs of closed infinite-dimensional subspaces of $\mathcal{H}$ is defined by

$$
\begin{aligned}
& \operatorname{Fred}^{2}(\mathcal{H}):=\{(\lambda, \mu) \mid \operatorname{dim} \lambda \cap \mu<\infty \text { and } \lambda+\mu \subset \mathcal{H} \text { closed } \\
& \text { and } \operatorname{dim} \mathcal{H} /(\lambda+\mu)<\infty\} .
\end{aligned}
$$

Remark A.1. (a) The property that $\lambda+\mu$ is closed in $\mathcal{H}$ in the preceding definition of a Fredholm pair $(\lambda, \mu)$ is very important for applications and often indispensable for establishing the finite codimension of the sum $\lambda+\mu$. On the other hand, it follows from the finite codimension in the following way: since $\mathcal{H} /(\lambda+\mu)$ has finite dimension, we can find $v_{1}, \ldots, v_{n} \in \mathcal{H}$ whose classes in $\mathcal{H} /(\lambda+\mu)$ form a basis. The linear span $h$ of $v_{1}, \ldots, v_{n}$ is then an algebraic complement of $\lambda+\mu$ in $\mathcal{H}$. Consider the map $\Psi: \lambda \oplus \mu \oplus h \rightarrow \mathcal{H}$ with $\Psi\left(u, u^{\prime}, v\right):=u+u^{\prime}+v$. Since $\Psi$ is linear, surjective, and (by definition) continuous, we have that $\Psi$ is open (again, according to the open mapping principle). It follows that $\mathcal{H} \backslash(\lambda+\mu)=\Psi(\lambda \oplus \mu \oplus h \backslash \lambda \oplus \mu \oplus\{0\})$ is open.

(b) Be aware that in spite of the strength of the open-mapping argument, it can not be applied to show that any subspace $W$ of finite codimension $\operatorname{dim} \mathcal{H} / W<\infty$ is closed. Of course, one could once again construct a bounded surjective operator $\Phi: \mathcal{H} \oplus W \rightarrow \mathcal{H}$, say by $\Phi(u, v):=u+v$. But, in general, $\mathcal{H} \backslash W$ can not be obtained as the image of an open subset of $\mathcal{H} \oplus W$ by applying $\Phi$. Certainly. $\mathcal{H} \backslash W \neq \Phi(\mathcal{H} \oplus W \backslash \mathcal{H} \oplus\{0\})$. Actually, the kernel $\operatorname{ker}(f)$ of any unbounded linear functional provides a counter example. It is a space of codimension 1 , but it is not closed since closed $\operatorname{ker}(f)$ would imply continuity of $f$ in 0 and hence everywhere.

(c) However, given a closed subspace $W \subset \mathcal{H}$ of finite codimension, clearly we have that any subspace $V$ with

$$
W \subset V \subset \mathcal{H}
$$

is closed and of finite codimension.

We are going to prove the following characterization of Lagrangian Fredholm pairs.

Proposition A.2. Let $\lambda, \mu \in \operatorname{Lag}(\mathcal{H})$ and let $\pi_{\lambda}, \pi_{\mu}$ denote the orthogonal projections of $\mathcal{H}$ onto $\lambda$ respectively $\mu$. Then $\pi_{\lambda}+\pi_{\mu}$ is a Fredholm operator, if and only if $(\lambda, \mu)$ is a Fredholm pair. 
Remark A.3. Our proposition is inspired by [3], Lemma 2.6 which states the following: Let $\lambda, \mu$ be a pair of closed infinite-dimensional subspaces with infinite-dimensional orthogonal complements. Let $\pi_{\lambda}$, $\pi_{\mu}$ denote the orthogonal projections. Then

$$
(\lambda, \mu) \in \operatorname{Fred}^{2}(\mathcal{H}) \Longleftrightarrow \pi_{\lambda}-\pi_{\mu} \in \operatorname{Fred}(\mathcal{H})
$$

Note that there is a minor gap in the original proof of Lemma 2.6 which easily can be filled. We shall indicate below the necessary changes in the proof of our present proposition for establishing Lemma 2.6 for pairs of Lagrangian subspaces.

On this occasion we should like to point to a flaw in Corollary 2.7 of the quoted paper where it was, erroneously, claimed that a pair of two closed infinite-dimensional subspaces $(\lambda, \mu) \in \operatorname{Fred}^{2}(\mathcal{H})$, if and only if the sum $\pi_{\lambda}+\pi_{\mu}$ of the orthogonal projections is of the form $I d+$ compact. For a Fredholm pair $(\lambda, \mu)$, however, the sum $\pi_{\lambda}+\pi_{\mu}$ is not always of the form $I d+$ compact operator. Actually, we have many examples where the operator norm $\left\|\pi_{\lambda}+\pi_{\mu}\right\|$ of the sum can be made arbitrarily small. But if $\pi_{\lambda}+\pi_{\mu}$ is of the form $I d+$ compact operator, then the norm is always $\geq 1$.

Of course, in some cases it is of such form. In particular, the sum of the orthogonal projections is always of the form $I d+$ compact when $\lambda$ denotes the Cauchy data space of a Dirac operator on a compact smooth Riemannian manifold with boundary (i.e. the range of the Calderón projector) and $\mu$ denotes its orthogonal complement or, more generally, the kernel of any other pseudodifferential projection with the same principal symbol as the Calderón projection. Such pairs appear typically in the treatment of elliptic boundary value problems belonging to the Grassmannian of generalized Atiyah-Patodi-Singer projections, whereas more general elliptic boundary value problems lead to arbitrary Fredholm pairs $(\lambda, \mu)$, though still with $\lambda$ fixed as the Cauchy data space (see [4] for both types of global elliptic boundary problems).

To prove our proposition we shall use the following lemma.

Lemma A.4. Let $\lambda, \mu \in \operatorname{Lag}(\mathcal{H})$ and $(\lambda, \mu) \in \operatorname{Fred}^{2}(\mathcal{H})$. Assume that $\lambda, \mu$ are transversal (i.e., $\lambda \cap \mu=\{0\}$ ). Then the operator $\pi_{\lambda}+\pi_{\mu}$ : $\mathcal{H} \rightarrow \mathcal{H}$ is surjective. (Hence, $\pi_{\lambda}+\pi_{\mu}$ is an isomorphism).

Proof. Given a $w \in \mathcal{H}$, we have to find $z \in \mathcal{H}$ such that $\left(\pi_{\lambda}+\pi_{\mu}\right) z=w$. Then, since $\lambda \cap \mu=\{0\}$, there exists an operator

$$
A: \lambda^{\perp} \rightarrow \lambda
$$


with $J \circ A$ self-adjoint, such that

$$
\mu=\left\{x+A x \mid x \in \lambda^{\perp}\right\} .
$$

Now any $w \in \mathcal{H}=\lambda+\mu(=\mu+\lambda)$ can be written as

$$
w=a+A(a)+J(b) \quad \text { with } a, b \in \lambda^{\perp} .
$$

Putting

$$
z:=(a+A a)+J(v+A v) \in \mathcal{H}=\mu \oplus \mu^{\perp} \quad \text { with } v:=b+J A a \in \lambda^{\perp}
$$

we obtain $\pi_{\mu}(z)=a+A(a)$. Moreover,

$$
\begin{aligned}
z & =a+A a+J(b+J A a+A b+A J A a) \\
& =a++J b+J A b+J A J A a \\
& =a+(J \circ A)^{2}(a)+(J \circ A)(b)+J(b) .
\end{aligned}
$$

We notice that the first three summands all belong to $\lambda^{\perp}$ and the fourth to $\lambda$. That yields $\pi_{\lambda}(z)=J(b)$. Hence $\pi_{\lambda}+\pi_{\mu}$ is surjective.

Remark A.5. (a) Clearly, for a Fredholm pair $(\lambda, \mu)$ of transversal Lagrangian subspaces we have

$$
(\lambda+\mu)^{\perp}=\lambda^{\perp} \cap \mu^{\perp}=J(\lambda) \cap J(\mu)=J(\lambda \cap \mu)=\{0\},
$$

hence $\lambda+\mu=\mathcal{H}$ by closedness of $\lambda+\mu$.

(b) The Lemma remains valid, if we replace the sum $\pi_{\lambda}+\pi_{\mu}$ by the difference $\pi_{\lambda}-\pi_{\mu}$. In the proof we just replace $z$ by

$$
z^{\prime}:=-a-(J \circ A)^{2}(a)+J \circ A(b)+J(b) .
$$

Then $\left(\pi_{\lambda}-\pi_{\mu}\right)\left(z^{\prime}\right)=w$ for $w=a+A(a)+J(b) \in \lambda+\mu=\mathcal{H}$. Correspondingly, the proof of Proposition A.2 can be suitably modified to give the same result for $\pi_{\lambda}-\pi_{\mu}$ instead of $\pi_{\lambda}+\pi_{\mu}$, i.e. an alternative and more geometrical proof of [3], Lemma 2.6, for Lagrangian subspaces.

(c) The Lemma can be reformulated for general Fredholm pairs, i.e. not necessarily Lagrangian subspaces. Of course, we will not have $P \pm \pi_{\mu}: \mathcal{H} \rightarrow \mathcal{H}$ surjective even if $\lambda, \mu$ transversal. But we can establish $P \pm \pi_{\mu}: \mathcal{H} \rightarrow \lambda+\mu$ surjective by a similar argument.

Proof of Proposition A.2. Let $\pi_{\lambda}+\pi_{\mu}$ be a Fredholm operator. Then $\pi_{\lambda}(x)+\pi_{\mu}(x)=0$ implies

$$
\left(x, \pi_{\lambda}(x)\right)=\left(x,-\pi_{\mu}(x)\right)=-\left\|\pi_{\mu}(x)\right\|^{2}=\left\|\pi_{\lambda}(x)\right\|^{2} .
$$


Hence $\pi_{\lambda}(x)=\pi_{\mu}(x)=0$, which shows that

$$
k e r\left(\pi_{\lambda}+\pi_{\mu}\right)=\lambda^{\perp} \cap \mu^{\perp}=J(\lambda \cap \mu) .
$$

Since $\left(\pi_{\lambda}+\pi_{\mu}\right)(\mathcal{H}) \subset \lambda+\mu$, and range $\left(\pi_{\lambda}+\pi_{\mu}\right)$ is closed and of finite codimension, so $\lambda+\mu$ must be closed and also of finite codimension (as argued before). Hence $(\lambda, \mu)$ is a Fredholm pair.

Now we prove the opposite direction: if $(\lambda, \mu)$ is a Fredholm pair, then $\pi_{\lambda}+\pi_{\mu}$ is a Fredholm operator. Let $w \in \lambda+\mu$ and $w \perp(\lambda \cap \mu)$. Then from Lemma A.4, we have $z \in \lambda+\mu$ such that

$$
z \perp(\lambda \cap \mu) \quad \text { and } \quad \pi_{\lambda}(z)+\pi_{\mu}(z)=w .
$$

This follows by replacing the total Hilbert space $\mathcal{H}$ by $\mathcal{H}^{\prime}:=(\lambda \cap \mu)^{\perp} \cap$ $(\lambda+\mu)$, the first subspace $\lambda$ by $(\lambda \cap \mu)^{\perp} \cap \lambda$, and the second subspace $\mu$ by $(\lambda \cap \mu)^{\perp} \cap \mu$.

Note that in the new Hilbert space $\mathcal{H}^{\prime}$ the two orthogonal projections onto $\lambda \cap(\lambda \cap \mu)^{\perp}$ and $\mu \cap(\lambda \cap \mu)^{\perp}$ coincide with the projections $\pi_{\lambda}$ and $\pi_{\mu}$, respectively.

Now let $w \in \lambda+\mu$ be decomposed as

$$
w=w_{0}+w_{1}, \quad w_{0} \in \lambda \cap \mu, w_{1} \in(\lambda \cap \mu)^{\perp},
$$

and put $z=z_{0}+\frac{1}{2} w_{1}$, where $z_{0}$ satisfies

$$
\pi_{\lambda}\left(z_{0}\right)+\pi_{\mu}\left(z_{0}\right)=w_{0} .
$$

Such $z_{0}$ exists according to Lemma A.4 and the preceding argument. Then we have

$$
\pi_{\lambda}(z)+\pi_{\mu}(z)=\pi_{\lambda}\left(z_{0}+\frac{1}{2} w_{1}\right)+\pi_{\mu}\left(z_{0}+\frac{1}{2} w-1\right)=w_{0}+w_{1}=w
$$

which shows

$$
\left(\pi_{\lambda}+\pi_{\mu}\right)(\lambda+\mu)=\lambda+\mu=\left(\pi_{\lambda}+\pi_{\mu}\right)(\mathcal{H}) .
$$

Hence we proved that the image of $\pi_{\lambda}+\pi_{\mu}$ is closed and of finite codimension. In fact, it coincides with $\lambda+\mu$. Clearly, the kernel $\operatorname{ker}\left(\pi_{\lambda}+\pi_{\mu}\right)=J(\lambda \cap \mu)$ is finite-dimensional.

\section{REFERENCES}

[1] B. BoJARSKI, The abstract linear conjugation problem and Fredholm pairs of subspaces, in "In Memoriam I.N. Vekua", Tbilisi Univ, Tbilisi, 1979, pp. 45-60 (Russian).

[2] B. Booss-BavnbeK and K. Furutani, The Maslov index - a functional analytical definition and the spectral flow formula, Tokyo J. Math. 21 (1998), $1-34$. 
[3] B. Booss-BavnbeK AND K. P. WoJcIECHOWSKI, Desuspension of splitting elliptic symbols, II, Ann. Glob. Analysis and Geometry 4 (1986), 349-400.

[4] -, -, "Elliptic Boundary Problems for Dirac Operators", Birkhäuser, Boston, 1993.

[5] J. BRÜNING AND M. LESCH, On boundary value problems for Dirac operators, Berlin, 1999 (in preparation).

[6] S. E. Cappell, R. Lee, and E. Y. Miller, On the Maslov index, Comm. Pure Appl. Math. 47 (1994), 121-186.

[7] -, -, -, Selfadjoint elliptic operators and manifold decompositions Part I: Low eigenmodes and stretching, Comm. Pure Appl. Math. 49 (1996), 825-866. Part II: Spectral flow and Maslov index, Comm. Pure Appl. Math. 49 (1996), 869-909. Part III: Applications to Casson's invariant (announced).

[8] R. G. Douglas AND K. P. Wojciechowski, Adiabatic limits of the $\eta$-invariants. The odd-dimensional Atiyah-Patodi-Singer problem, Comm. Math. Phys. 142 (1991), 139-168.

[9] M. DE Gosson, The structure of $q$-symplectic geometry, J. Math. Pures Appl. 71 (1992), 429-453.

[10] L. HÖRMANDER, Pseudo-differential operators and non-elliptic boundary problems, Ann. of Math. 83 (1966), 129-209.

[11] -, Fourier integral operators, I, Acta Math. 127 (1971), 79-183.

[12] L. NicolaEsCU, The Maslov index, the spectral flow, and decomposition of manifolds, Duke Math. J. 80 (1995), 485-533.

[13] R. S. Palais, Homotopy type of infinite dimensional manifolds, Topology 5 (1966), 1-16.

[14] J. PhILLIPS, Self-adjoint Fredholm operators and spectral flow, Canad. Math. Bull. 39 (1996), 460-467.

[15] A. PLIŚ, A smooth linear elliptic differential equation without any solution in a sphere. Comm. Pure Appl. Math. 14 (1961), 599-617.

[16] J. V. RALSTON, Deficiency indices of symmetric operators with elliptic boundary conditions, Comm. Pure Appl. Math. 23 (1970), 221-232.

[17] J. Robbin AND D. Salamon, The Maslov index for paths, Topology 32 (1993), 827-844.

[18] R. C. Swanson, Fredholm intersection theory and elliptic boundary deformation problems, I, J. of Diff. Equations 28 (1978), 189-201.

[19] T. YOSHIDA, Floer homology and splittings of manifolds, Ann. of Math. 134 (1991), 277-323.

INSTITUT FOR MATEMATIK OG FYSIK, ROSKILDE UNIVERSITY, DK-4000 ROSKILDE, DENMARK

E-mail address: booss@mmf.ruc.dk

Department of Mathematics, Science University of Tokyo, NodaSHI, CHIBA-KeN 278-8510 JAPAN

E-mail address: furutani@ma.noda.sut.ac.jp 
227,92 'Computeisimulezing og fysik'

af: Pez H.Hansen. Sreffen Holni,

Pete: Naibom, Mads K. Dall Peterse!:

Pernille Postgaara, 'homas B. Schtocie: zvar P. Zeck

Vejlede: : Peder Voetmann Ch:iswianse:

Liste over tidijigere uäkomne tekster tilsendes geme. Henvendelse herom kan ske til IMFUFA's sekretariat

tif. 46742263
228,92 Teknologi og histoite:

Fire aztikle: af :

Mogens Niss, ens iloyzup, ib nilie-sen, Hans Hecal

229/92 "Masse: af information uden becycining En diskussion af informationstẹo:ien i TOL Nozetianceis: "Mask Vercen" og en skiise ill et al cernativ basse.t. pá ancienordens $k j b e i n e t z k$ og senionik. af: Soien $B_{i}$-iei

230/92 "Vinklens treceling - et klassisk problem"

et matemac isk projekt af

Karen Bitkelund, Bjoin Christensen Vejlede: : cohnny Ozzesen

231A/92 "Elektzonciffusion i siliciun - en matemaisk mociel"

af: iesper Voetmann, Karen Birkelunc, wette Olufsen, Ole voller Nielsen Vejleciere: cohnny oriesen, F.B.Hansen

231B;92 "Elektionciffusion i siliciun - en matemarisk model" Kildetekste:

af: vespe: Voetuann, Karen Bistirelur. Viette OIufsen, Ule Mulle: kielsen Vejlecieze: iohnny orzesen, I. B. ilanse:

232/92 "Undersogelse oii den simulaane opäagelse af eneigiens bevarelse og isiacies olt de af Nayer, Coläing, voule og llelaholcz udforte arbejce:"

af: I.Azlech, G.I.Dybkjæ: M.T.Osie.gö Vejlede: : Dorthe Posselt

233 ,92 "The effect of age-depenone host mortality on the dynamics of an enciemic disease and

Instabilicy in an SER-nodel wizh agedepencent susceptibility

by: Viggo Ancieasen

234/92 "THE FUNCTIONAL DETERMINANT OF A FOUR-DZ̈.UNSIONAL BOUNDARY VALUE FROBLEM"

by: Thomas P. Branson and Peter B. Gilkey

235,92 OVERFLADESTRUKTUR OG POREUDVIKLING AF KOKS

- Modul 3 fysik projekt -

af: Thomas Jessen

226/92 "METHODS AND MODELS FOR ESTIMATING THE GLOBAL CIRCULATION OF SELECTED EMISSTONS FROM ENERC? CONVERSION ${ }^{*}$

by: Bent Sorensen 
$236 a / 93$ INTRODUKTION TIL KVANTE

HALL EFFEKTEN

af: Anja Boisen, Peter Boggild

Vejleder: Peder Voetmann Christiansen

Erland Brun Hansen

$236 \dot{b} / 93$ STRGMSSAMMENBRUD AF KVANTE

HALL EFFEKTEN

af: Anja Boisen, Peter Boggild

Vejleder: Peder Voetmann Christiansen Erland Brun Hansen

237/93 The wedderburn principal theorem and Shukla cohomology

af: Lars Kadison

$238 / 93$ SEMIOTIK OG SYSTEMEGENSKABER (2)

Vektorband og tensorer

af: Peder Voetmann Christiansen

$239 / 93$ Valgsystemer - Modelbygning og analyse

Matematik 2. modul

af: Charlotte Gjerrild, Jane Hansen.

Maria Hermannsson, Allan Jorgensen,

Ragna Clauson-Kaas, Poul Lützen

Vejleder: Mogens Niss

240/93 Patologiske eksempler.

Om sare matematiske fisks betydning for

den matematiske udvikling

af: Claus Dræby, Jørn Skov Hansen, Runa

Ulsee Johansen, Peter Meibom, Johannes

Kristoffer Nielsen

vejleder: Mogens Niss

241,93 FOTOVOLTAISK STATUSNOTAT 1

af: Bent Sørensen

242,93 Brovedligeholdelse - bevar mig vel

Analyse af vejdirektoratets model for

optimering af broreparationer

af: Iinda Kyndlev, Kare Fundal, Kamma

Tulinius, ivar Zeck

Vejleder: Jesper Larsen

243/93 TANKEEKSPERIMENTER I FYSIKKEN

Et 1.modul fysikprojekt

af: Karen Birkelund, Stine Sofia Korremann

Vejleder: Dorthe Posselt

244,93 RADONTRANSFORMATIONEN Oq dens anvendelse

i CT-scanning

Projektrapport

af: Trine Andreasen, Tine Guldager Christiansen,

Nina Skov Hansen og Christine Iversen

Vejledere: Gestur Olafsson og jesper Larsen

$245 a+b$

193 Time-of-Flight målinger på krystallinske

halvledere

Specialerapport

af: Iinda Szkotak Jensen og Iise Odgaard Gade

Vejledere: Petr Viscor og Niels Boye olsen

246/93 HVERDAGSVIDEN OG MATEMATIK

- LEREPROCESSER I SKOLEN

af: Lena Lindenskov, Statens Humanistiske

Forskningsråd, RUC, IMFUFA
247,93 UNZVERSAL LOW TEMPERATURE AC CONDUCTEVITY OE MACROSCOPICAILY DISORDERED NON-METALS

by: Jeppe C. Dyre

$248 / 93$ DIRAC OPERATORS AND MANIFOLDS WITH BOUNDARY

by: B. Booss-Bavnbek, K.P.Wojciechowsk:

249/93 Perspectives on Teichmüller and the zahresbericht Addendum to Schappache:. Scholz, et al.

by: B. Booss-Bavnbek

With comments by W.Abikoff, I.Ahlfors, J.Cerf, P.J.Davis, W.Fuchs, F.P.Gardine:J.Jost, j.-P.Kahane, R.Lohan, L.Lo:ch, J.Radkau and T.Söderquist

250/93 EULER OG BOLZANO - MATENATISK ANALYSE SET I E:VIDENSKABSTEORETJSK PERSPEKTTV

Projektrapport af: Anja Juul, Lone Michelser. Tomas hojgåna .jensen

Vejleder: Stig Andur Pedersen

$251 \mid 93$ Genotypic Proportions in Bybrid Zones by: Freddy Bugge Christiansen, Viggo Andreasen and Ebbe Thue Poulsen

$252 \mid 93$ MODELLERING AF TILFALDTGE FAWOMENER

Projektrapport af: Birthe Friis, Lisbeth Belmgaard Kristina Charlotte Jakobsen, Marina Mosbak Johonnessen, Lotte Ludvigsen, Mette Bass Nielsen

253/93 - Kuglepaking

Teori og model

af: Lise Arleth, Käre Fundal, Wils Kruse

Vejleder: Mogens Niss

254/93 Regressionsonalyse

Materiale til et statistikkursus

af: Jorgen Larsen

$255 / 93$ TID \& BETINGET UAFHANGIGHED

af: Peter Harremoës

256/93 Determination of the Frequency Dependent Bulk Modulus of Liquids Using a Piezoelectric Spherical Shell (Preprint)

by: T. Christensen and N.B.OZsen

257/93 Modellering af dispersion $i$ piezoelektriske kerconikker

af: Pernille Postgaard, Jannik Rasmussen, Christina Specht, Mikko ostergäd

Vejleder: Tage Christensen

258/93 Supplerende karsusmateriale til "Lineare strukturer fra algebra og analyse"

af: Mogens Bron Beefelt

259/93 STUDIES OF AC BOPPING CONDUCTION AT LOW TEMPERATURES

by: Jeppe C. Dyre

260/93 PARTITIONED MANIFOLDS AND INVARIANTS IN DTMENSTONS 2,3, AWD 4

by: B. Booss-Baunbek, K.P. Wojciechouski 
$261: 93$ OPGAVESAMLING

Bredde-kursus i Fysik

Eksamensopgaver fra 1976-93

262/93 Separability and the Jones Polynomial

by: Lars Kadison

263/93 Supplerende kursusmateriale til "Lineære strukturer fra algebra oq analyse" II

af: Mogens Brun Heefelt

$264: 93$ FOTOVOLTAISK STATUSNOTAT 2 af: Bent sorensen

265/94 SPHERICAL FUNCTIONS ON ORDERED SYMMETRIC SPACES

To Sigurdur Helgason on his sixtyfifth birthday

by: Jacques Faraut, Joachim Hilgert and Gestur Olafsson

266/94 Kommensurabilitets-oscillationer $i$ laterale supergitre

Fysikspeciale af: Anja Boisen, Peter Boggild, Karen Birkelund

Vejledere: Rafael Taboryski, Poul Erik Iindelof, Peder Voetmann Christiansen

$267 / 94$ Kom til kort med matematik på

Eksperimentarium - Et forslag til en

opstilling

af: Charlotte Gjerrild, Jane Hansen

Vejleder: Bernhelm Booss-Bavnbek

268/94 Life is like a sewer...

Et projekt om modellering af aorta via en model for strøming i kloakrør

af: Anders Marcussen, Anne C. Nilsson, Lone Michelsen, Per M. Hansen

Vejleder: Jesper Larsen

269/94 Dimensionsanalyse en introduktion metaprojekt, fysik

af: Tine Guläager Christiansen,

Ken Andersen, Nikolaj Hermann,

Jannik Rasmussen

Vejleder: Jens Hojgaard Jensen

270/94 THE IMAGE OF THE ENVELOPING ALGEBRA AND IRREDUCIBIIITY OF INDUCED REPRESENTATIONS OF EXPONENTIAL IIE GROUPS

by: Jacob تacobsen

271/94 Matematikken i Fysikken.

Opaaget eller opfundet

NAT-BAS-2rojekt

vejleder: Jens Højgaard Jensen
272/94 Tradition og fornyelse

Det praktiske elevarbejde $i$ grmnasiets fysikundervisning, 1907-1988

af: Kristian Hoppe og Jeppe Guldager

Vejledning: Karin Beyer og Nils Hybel

273/94 Model for kort- og mellemdistancelob verifikation af model

af: Lise Fabricius Christensen, Helle Pilemann, Bettina Sorensen

Vejleder: Mette Olufsen

274/94 MODEL 10 - en matematisk model af intravenose anæs tetikas farmakokinetik.

3. modul matematik, forår 1994

af: Trine Andreasen, Bjorn Christensen, Christine Green, Anja Skjoldborg Hansen. Lisbeth Helmgaard

Vejledere: Viggo Andreasen \& Jesper Larsen

275/94 Perspectives on Teichmiller and the Jahresbericht and Edition

by: Bernhelm Booss-Bavnbek

276/94 Dispersionsmodellering Projektrapport 1. modul

af: Gitte Andersen, Rehannah Borup, Lisbeth Friis, Per Gregersen, Kristina Vejro

Vejleder: Bernhelm Booss-Bavnbek

277/94 PROJEKTARBEJDSPEDAGOGIK - Om tre tolkninger af problemorienteret projektarbejde

af: Claus Flensted Behrens, Frederik Voetmann Christiansen, Jorn Skov Hansen, Thomas Thingstrup

Vejleder: Jens Højgaard Jensen

278/94 The Models Underlying the Anaesthesia Simulator Sophus

by: Mette Olufsen(Math-Tech), Finn Nielsen (RISO National Laboratory), Per Foge Jensen (Herlev University Hospital), Stig Andur Pedersen (Roskilde University)

279/94 Description of a method of measuring the shear modulus of supercooled liquids and a comparison of their thermal and mechanical response functions.

af: Tage Christensen

280/94 A Course in Projective Geometry by Lars Kadison and Matthias $T$. Kromann

281/94 Modellering af Det Cardiovaskulare System med Neural Pulskontrol

Projektrapport udarbejdet af:

Stefan Frello, Runa Ulsøe Johansen, Michael Poul Curt Hansen, Klaus Dahl Jensen

Vejleder: Viggo Andreasen

282/94 Parallelle algoritmer

af: Erwin Dan Nielsen, Jan Danielsen, Niels Bo Johansen 
283/94 Grænser for tilfældighed

(en kaotisk talgenerator)

af: Erwin Dan Nielsen og Niels Bo Johansen

284/94 Det er ikke til at se det, hvis man ikke lige ve' det

Gymnasiematematikkens begrundelsesproblem

En specialerapport af Peter Hauge Jensen

og Linda Kyndlev

Veileder: Mogens Niss

285/94 Slow coevolution of a viral pathogen and its diploid host

by: Viggo Andreasen and Freddy B. Christiansen

286/94 The energy master equation: A low-temperature approximation to Bassler's random walk model by: Jeppe $C$. Dyre

287/94 A Statistical Mechanical Approximation for the Calculation of Time Auto-Correlation Functions by: Jeppe $C$. Dyre

288/95 PROGRESS. IN WIND ENERGY UTILIZATION by: Eent Sorensen

289/95 Universal Time-Dependence of the Mean-Square Displacement in Extremely Rugged Energy Landscapes with Equal Minima

by: Jeppe C. Dyre and Jacob Jacobsen

290/95 Modellering af uregelmassige bolger

Et 3 .modul matematik projekt

af: Anders Marcussen, Anne Charlotte Nilsson, Lone Michelsen, Per Morkegaard Hansen

Vejleder: Jesper Larsen

291/95 1st Annual Report from the project LIFE-CYCLE ANALYSIS OF THE TOTAL DANISH ENERGY SYSTEM

an example of using methods developed for the $O E C D / I E A$ and the US/EU fuel cycle externality study by: Bent Serensen

292/95 Fotovoltaisk Statusnotat 3

af: Bent Serensen

293/95 Geometridiskussionen - hvor blev den af?

af: Lotte Ludvigsen \& Jens Frandsen

Vejledir: Anders Madsen

294/95 Universets udvidelse et metaprojekt

Af: Jesper Duelund og Birthe Friis

Vejleder: Ib Lundgaard Rasmussen

295/95 A Review of Mathematical Modeling of the Controled Cardiovascular System

By: Johnny T. Ottesen
296/95 RETIKULER den klassiske mekanik af: Peder Voetmann Christiensen

$297 / 95$ A fluid-dynamical model of the aorta with bifurcations

by: Mette Olufsen ano johnny Ottesen

298/95 Mordet på Schrödingers kat - et metaprojekt om to fortolkninger af kvantemekanikken

af: Maria Hermannsson, Sebastian Horst, Christina Specht

Vejledere: Jeppe Dyre og Peder Voetmann Christiansen

299/95 ADAM under figenbladet - et kig på en samfundsvidenskabelig matematisk model

Et matematisk modelprojekt

af: Claus Dræby, Michael Hansen, Tomas Højgård Jensen Vejleder: Jørgen Larsen

$300 / 95$ Scenarios for Greenhouse Warming Mitigation by: Bent Sorensen

301/95 TOK Modellering af træers vækst under pảvirkning af ozon

af: Glenn Moller-Holst, Marina Johannessen, Birthe Nielsen og Bettina Sorensen

Vejleder: Jesper Larsen

302/95 KOMPRESSORER - Analyse af en matematisk model for aksialkompressorer

Projektrapport sf: Stine Beggild, Jakob. Hilmer, Pernille Postgaard

Vejleder: Viggo Andreasen

303/95 Masterlignings-modeller af Glasovergangen Termisk-Mekanisk Relaksation Specialerapport udarbejdet af :

Johannes K. Nielsen, Klaus Dahl Jensen

Vejledere: Jeppe C. Dyre, Jorgen Larsen

304a/95 STATISTIKNOTER Simple binomialfordelingsmodeller af: Jørgen Larsen

304b/95 STATISTIKNOTER simple normalfordelingsmodeller af: Jorgen Larsen

304c/95 STATISTIKNOTER Simple Poissonfordelingsmodeller af: Jergen Larsen

304d/95 STATISTIKNOTER Simple multinomialfordelingsmodeller af: Jørgen Larsen

304e/95 STATISTIKNOTER Mindre matematisk-statistisk opslagsvirk indeholdende bl.a. ordforklaringer, resuméer og tabeller af: Jorgen Larsen 
The Maslov Index:

A Functional Analytical Definition

And The Spectral Flow Formula

By: B. Booss-Bavnbek, K. Furutani

306/95 Goals of mathematics teaching

Preprint of a chapter for the forthcomming-International Handbook of Mathematics Education (Alan J.Bishop, ed)

By: Mogens Niss

307/95 Habit Formation and the Thirdness of Signs

Presented at the semiotic symposium

The Emergence of Codes and Intensions as a Basis of Sign Processes

By: Peder Voetmann Christiansen

308/95 Metaforer i Fysikken

af: Marianne Wilcken Bjerregaard, Frederik Voetmann Christiansen,

Jorn Skov Hansen, Klaus Dahl Jensen

Ole Schmidt

Vejledere: Peder Voetmann Christiansen og Petr Viscor

309/95 Tiden og Tanken

En undersøgelse af begrebsverdenen Matematik udfort ved hjælp af en analogi med tid

af: Anita Stark og Randi Petersen

Vejleder: Bernhelm Booss-Bavnbek

310/96 Kursusmateriale til "Lineare strukturer fra algebra og analyse" (E1)

af: Mogens Brun Heefelt

311/96 2nd Annual Report from the project LIFE-CYCLE ANALYSIS OF THE TOTAL DANISH ENERGY SYSTEM

by: Hélène Connor-Lajambe, Bernd Kuemmel, Stefan Krüger Nielsen, Bent Sørensen

312/96 Grassmannian and Chiral Anomaly

by: B. Booss-Bavnbek, K.P.Wojciechowski

313/96 THE IRREDUCIBILITY OF CHANCE AND THE OPENNESS OF THE FUTURE

The Logical Function of Idealism in Peirce's Philosophy of Nature

By: Helmut Pape, University of Hannover

314/95 Feedback Regulation of Mammalian

Cardiovascular system

By: Johnny $\mathbf{T}$. Ottesen

315/96 "Rejsen til tidens indre" - Udarbejdelse af

$a+b$ et manuskript til en fjernsynsudsendelse

+ manuskript

af: Gunhild Hune og Karina Goyle

Vejledere: Peder Voetmann Christiansen og Bruno Ingemann
316/96 Plasmaoscillation i natriumlilynge:

Specialerapport af: Pezer Meibom, Mikio istersis: Vejledere: Jeppe Dyre \& Jorn Borggreer.

317/96 Poincaré og symplektiske algoritmer

ar: Ulla Rasmussen

Vejleder: Anders Madsen

318/96 Modelling the Respiratory System by: Tine Guldager Christiansen, Claus Dræby Supervisors: Viggo Andreasen, Michael Danielsen

319/96 Externality Estimation of Greenhouse Warming Impacts

by: Bent Sorensen

320/96 Grassmannian and Boundary Contribution to the -Determinant

by: K.P.Wojciechowski et al.

321/96 Modelkompetencer - udvikling og afprovning af et begrebsapparat

Specialerapport af: Nina Skov Hansen, Christine Iversen, Kristin Troels-Smith Vejleder: Morten Blomhoj

322/96 OPGAVESAMLING

Bredde-Kursus i Fysik 1976 - 1996

$323 / 96$ Structure and Dynamics of Symmetric Diblock Copolymers

PhD Thesis

by: Christine Maria Papadakis

$324 / 96$ Non-1inearity of Baroreceptor Nerves by: Johnny T. Ottesen

325/96 Retorik eller realitet ?

Anvendelser af matematik i det danske Gymnasiums matematikundervisning $i$ perioden $1903-88$

Specialerapport af Helle Pilemann Vejleder: Mogens Niss

326/96 Bevisteori

Eksemplificeret ved Gentzens bevis for konsistensen af teorien om de naturlige tal

af: Gitte Andersen, Lise Mariane jeppesen, Klaus Frovin Jorgensen, Ivar Peter Zeck

Vejledere: Bernhelm Booss-Bavnibk og Stig Andur Pedersen

327/96 NON-LINEAR MODELLING OF INTEGRATED ENERGY SUPPLY AND DEMAND MATCHING SYSTEMS by: Bent Sørensen

328/96 Calculating Fuel Transport Emissions by: Bernd Kuemmel 
$329 / 96$ The dynamics of cocirculating influenza strains conferring partial cross-immunity and

A model of influenza A drift evolution

by: Viggo Andreasen, Juan Lin and

Simon Levin

330/96 LONG-TERM INTEGRATION OF PHOTOVOLTAICS INTO THE GLOBAL ENERGY SYSTEM

by : Bent Sørensen

\section{1/96 Viskese fingre}

Specialerapport af:

Vibeke orlien og Christina Specht

Vejledere: Jacob M. Jacobsen og Jesper Larsen

332/97 ANOMAL SWELLING AF LIPIDE DOBBELTLAG

Specialerapport af:

Stine Sofia Korremann

Vejleder: Dorthe Posselt

333/97 Biodiversity Matters

an extension of methods found in the literature on monetisation of biodiversity

by: Bernd Kuemmel

334/97 IIFE-CYCLE ANALYSIS OF THE TOTAL DANISH ENERGY SYSTEM

by: Bernd Kuemmel and Bent Sorensen

335/97 Dynamics of Amorphous Solids and Viscous Liquids by: Jeppe C. Dyre

336/97 PROBLEM-ORIENTATED GROUP PROJECT WORK AT ROSKILDE UNIVERSITY

by: Kathrine Legge

337/97 Verdensbankens globale befolkningsprognose

- et projekt om matematisk modeliering

af: Jorn Chr. Bendtsen, Kurt Jensen, Per Pauli Petersen

Vejleder: Jorgen Larsen

338/97 Kvantisering af nanolederes elektriske

ledningsevne

Forste modul fysikprojekt

af: Soren Dam, Esben Danielsen, Martin Niss, Esben Friis Pedersen, Frederik Resen Steenstrup Vejleder: Tage Christensen
339/97 Defining Discipline by: Wolfgang Coy

340/97 Prime ends revisited - a geometric point of view -

by: Carsten Lunde Petersen

341/97 Two chapters on the teaching. learning and assessment of geometry

by Mogens Niss

342/97 LONG-TERM SCENARIOS FOR GLOBAL ENERGY DEMAND AND SUPPLY

A global clean fossil scenario discussion paper prepared by Bernd Kuemmel

Project leader: Bent Sorensen

343/97 IMPORT/EKSPORT-POLITIK SOM REDSKAB TIL OPTIMERET UDNYTTELSE AF EL PRODUCERET PÁ VE-ANLFG

af: Peter Meibom, Torben Svendsen, Bent Sorensen

344/97 Puzzles and Siegel disks

by Carsten Lunde Petersen

345/98 Modeling the Arterial System with Reference to an Anestesia Simulator

Ph.D. Thesis

by: Mette Sofie Olufsen

346/98 Klyngedannelse $i$ en hulkatode-forstøvningsproces

af: Sebastian Horst

Vejledere: Jorn Borggren, NBI, Niels Boye Olsen

$347 / 98$ Verificering af Matematiske Modeller

- en analyse af Den Danske Eulerske Model

af: Jonas Blomqvist, Tom Pedersen, Karen Timmermann, . Lisbet ohienschlager

Vejleder: Bernhelm Booss-Baunbek

348/98 Case study of the environmental permission procedure and the environmental impact assessment for power plants in Denmark

by: Stefan Krüger Nielsen

Project leader: Bent Sørensen

349/98 Tre rapporter fra FAGMAT - et projekt om tal og faglig matematik $i$ arbejdsmarkedsuddannelserne af: Lena Lindenskov og Tine Wedege

350/98 OPGAVESAMLING - Bredde-Kursus i Fysik 1976 - 1998 Erstatter teksterne $3 / 78,261 / 93$ og $322 / 96$

351/98 Aspects of the Nature and State of Research in Mathematics Education

by: Mogens Niss 
352/98 The Herman-Swiatec Theorem with applications

by: Carsten Lunde Petersen

353/98 Problemlesning og modellering $i$

en almendannende matematikundervisning

Specialerapport af: Per Gregersen og

Tomas Højgaard Jensen

Vejleder: Morten Blomhoj

354/98 A GLOBAL RENEWABLE ENERGY SCENARIO

by: Bent Sørensen and Peter Mejbom

$355 / 98$ Convergence of rational rays in

parameter spaces

by: Carsten Lunde Petersen and

Gustav Ryd

356/98 Terrænmodellering

Analyse af en matematisk model til konstruktion af terrænmodeller

Modelprojekt af: Thomas Frommelt,

Hans Ravnkjær Larsen og Arnold Skimminge

Vejleder: johnny Ottesen

357/98 Cayleys Problem

En historisk analyse of arbejdet med Cayley problem fra 1870 til 1918

Et matematisk videnskabsfagsprojekt af:

Rikke Degn. Bo Jakobsen. Bjarke K.W. Hansen,

Jesper $S$. Hansen, Jesper Udesen, Peter C. Wulff

Vejleder: Jesper Larsen

358/98 Modeling of Feedback Mechanisms which Control the Heart Function in a View to an Implementation in Cardiovascular Models

Ph.D. Thesis by: Michael Danielsen

359/99 Lona-Term Scenarios for Global Eneray Demand and Supply Four Global Greenhouse Mitigation Scenarios

by: Bent Sorensen

360/99 STMETRI I FYSIK

En Meta-projektrapport af: Martin Niss.

Ba Jakobsen \& Tune Bjarke Bonné

Vejleder: Peder Voetmann Christiansen

361/99 Symplectic Functional Analysis and Spectral

invariants

by: Bermhelm Booss-Baunbek, Kenro Eurutani

362/99 Er matematik en naturvidenskab? - en udspan-

ding of diskussionen

En videnskabsfagsprojekt-rapport af Martin Niss

Vejleder: Mogens Norgaard Olesen 